\title{
Ultracompact HII regions with extended emission: The case of G43.89-0.78 and its molecular environment
}

\author{
Eduardo de la Fuente, ${ }^{\star \star} \dagger$ Daniel Tafoya ${ }^{2}$, Miguel A. Trinidad ${ }^{3}$, Alicia Porras ${ }^{4}$, \\ Alberto Nigoche-Netro ${ }^{5}$, Simon N. Kemp ${ }^{5}$, Stanley E. Kurtz ${ }^{6}$, José Franco, ${ }^{7}$ \\ and Carlos A. Rodríguez-Rico ${ }^{3}$ \\ ${ }^{1}$ Departamento de Física, CUCEI, Universidad de Guadalajara, Blvd. Gral. Marcelino García Barragán 1421, Olímpica, 44430, Guadalajara, Jalis \\ ${ }^{2}$ Department of Space, Earth and Environment, Chalmers University of Technology, Onsala Space Observatory, 439 92 Onsala, Sweden. \\ ${ }^{3}$ Departamento de Astronomía, Universidad de Guanajuato, Apartado Postal 144, 36000, Guanajuato, Guanajuato, México. \\ ${ }^{4}$ Instituto Nacional de Astrofísica, Óptica y Electrónica, Luis E. Erro Número 1, 72840, Tonantzintla, San Andrés Cholula, Puebla, México. \\ ${ }^{5}$ Instituto de Astronomía y Meteorología, CUCEI, Universidad de Guadalajara, Av. Vallarta 2602, 44130, Guadalajara, Jalisco, México. \\ ${ }^{6}$ Instituto de Radioastronomía y Astrofísica, UNAM, Antigua Carretera a Pátzcuaro Numero 8701, 58089, Morelia, Michoacán, México. \\ ${ }^{7}$ Instituto de Astronomía, UNAM, Apartado Postal 70-264, CDMX, 04510, México
}

To be Published on Monthly Notices of the Royal Astronomical Society Main Journal. Accepted 2020 July 15. Received 2020 July 15; in original form 2020 June 10

\begin{abstract}
The Karl Jansky Very Large Array (VLA), Owens Valley Radio Observatory (OVRO), Atacama Large Millimetric Array (ALMA), and the infrared Spitzer observatories, are powerful facilities to study massive star formation regions and related objects such as ultra-compact (UC) H II regions, molecular clumps, and cores. We used these telescopes to study the UC H II region G43.89-0.78. The morphological study at arcminute scales using NVSS and Spitzer data shows that this region is similar to those observed in the bubble-like structures revealed by Spitzer observations. With this result, and including a physical characterization based on $3.6 \mathrm{~cm}$ data, we suggest G43.89-0.78 be classified as an UC H II region with Extended Emission because it meets the operational definition given in this paper comparing radio continuum data at 3.6 and $20 \mathrm{~cm}$. For the ultra-compact component, we use VLA data to obtain physical parameters at $3.6 \mathrm{~cm}$ confirming this region as an UC H II region. Using ALMA observations, we detect the presence of a dense $\left(2.6 \times 10^{7} \mathrm{~cm}^{-3}\right)$ and small $\left(\sim 2.0^{\prime \prime}\right.$; $0.08 \mathrm{pc}$ ) molecular clump with a mass of $220 \mathrm{M}_{\odot}$ and average kinetic temperature of $21 \mathrm{~K}$, located near to the UC H II region. In this clump, catalogued as G43.890-0.784, water masers also exist, possibly tracing a bipolar outflow. We discover in this vicinity two additional clumps which we label as G43.899-0.786 $\left(\mathrm{T}_{d}=50 \mathrm{~K} ; \mathrm{M}=11 \mathrm{M}_{\odot}\right)$, and G43.888-0.787 ( $\left.\mathrm{T}_{d}=50 \mathrm{~K} ; \mathrm{M}=15 \mathrm{M}_{\odot}\right)$.
\end{abstract}

Key words:

ISM: H II regions - ISM: Molecules — STARS: High mass — INFRARED: Gas-Dust — RADIO CONTINUUM: ISM - RADIO LINES: ISM

\section{INTRODUCTION}

The study of UC H II regions is very important to understand the high-mass star formation process because they trace regions with recently formed ionizing (OB-type) stars.

\footnotetext{
* Corresponding author e-mail: eduardo.delafuente@academicos.udg.mx

$\dagger$ Also HAWC Gamma-Ray National Laboratory (CONACyT), México. Research visit at Institute for Cosmic Ray Research (Feb. 2020), University of Tokyo, Kashiwa Campus, Japan.
}

The UC H II regions are often related to hot molecular cores and maser emission (e.g, Hofner \& Churchwell 1996; Garay \& Lizano 1999; Kurtz et al. 2000b; Churchwell 2002; de la Fuente et al. 2018, and references therein). They were identified by Wood \& Churchwell (1989) and Kurtz et al. (1994) as small (sizes $\leqslant 0.1 \mathrm{pc}$ ) and dense $\left(\gtrsim 10^{4} \mathrm{~cm}^{-3}\right)$ H II regions with high emission measures $\left(\geqslant 10^{7} \mathrm{pc} \mathrm{cm}^{-6}\right)$. The physical parameters of the ionized gas are determined by radio continuum $(\mathrm{RC})$ and radio recombination line (RRL) observations (e.g, Oster 1961; Mezger \& Henderson 1967; 
Schraml \& Mezger 1969; Dupree \& Goldberg 1970; Panagia \& Walmsley 1978).

There are five booms in the history of the topic: 1.- the original studies via single-dish observations (e.g, Mezger \& Henderson 1967; Ryle \& Downes 1967; Israel, Habing \& de Jong 1973, and references therein), 2.- the physical characterization for the first time via VLA interferometric observations (Wood \& Churchwell 1989; Kurtz et al. 1994), 3.observations (and studies) of extended emission (EE) at arcmin scales by de la Fuente 2007 (see Kurtz et al. 1999; Kim \& Koo 2001, 2002, 2003; Ellingsen, Shabala, \& Kurtz 2005; de la Fuente et al. 2009a,b, 2018, 2020), 4.- the discovery of time variation in the radio flux density (Franco-Hernández \& Rodriguez 2004; Gálvan-Madrid et al. 2008) and, 5.- the detection of hypercompact (HC) H II regions (Gaume et al. 1995; Sewiło et al. 2004, 2008; DePree, Wilner, \& Goss 2011). Reviews can be found in Kurtz (2000a); Churchwell (2002); de la Fuente et al. (2009a) and references therein.

In the morphological study performed by de la Fuente et al. (2020, Paper I hereafter), we found that extended radio continuum emission (EE) resembles bubble-like structures found in the Bubbling Universe revealed by Spitzer observations (e.g., Churchwell et al. 2006, 2007, 2009; Everett \& Churchwell 2010; Simpson et al. 2012, and references therein). Paper I also shows that for all the sources of the sample, except for G12.21-0.10 (G12.21 hereafter), the Spitzer MIPS emission at $24 \mu \mathrm{m}$ is saturated at the position of the UC component (the UC H II region). Hence, this MIPS band could be a good tracer of UC H II regions and especially those UC H II with EE (UC H II +EE regions) via visual inspection. Such infrared data could thus be an important complement to morphological studies by avoiding the spatial filtering of extended emission inherent to radiointerferometers.

Water maser emission is known to be a common - if not ubiquitous - phenomenon in star formation regions (e.g., Elitzur 1992; Garay \& Lizano 1999; Trinidad et al. 2003; Codella et al. 2004, and references therein). Nevertheless, various questions remain regarding the relation of the masers to the star formation process. Hofner \& Churchwell (1996) show that for UC H II regions with cometary morphologies, water masers are always located in clumps, near, but offset from, the cometary arc. These masers could be related to nearby, embedded young stellar objects rather than to the $\mathrm{H}$ II regions themselves.

G12.21 and G43.89-0.78 (G43.89 hereafter) are good candidates to study the nature of these water maser clumps because these two sources have larger separations between the masers and the H II region. Hence, the maser clump can be spatially resolved from ionized gas. In this context, the molecular clump with water masers observed in the UC component of the UC H II +EE region G12.21 was characterized as a hot molecular core via $\mathrm{NH}_{3}(2,2)$ and $(4,4)$ VLA observations by de la Fuente et al. (2018). These authors start their analysis with the detection of ${ }^{13} \mathrm{CS}(2-1)$ at the position of the water masers. They confirm that the masers arise within the molecular core instead of the H II region. A similar analysis was not performed for G43.89, hence, the origin of the masers remained unknown for this source. The purpose of the present paper is to provide such an analysis for G43.89 and further, elucidate the origin of water masers during the star formation process.
G43.89 (IRAS 19120+0917) is a cometary UC H II region with a diameter of $\sim 4^{\prime \prime}$ (Wood \& Churchwell 1989). Its distance is uncertain. Wink, Altenhoff, \& Mezger (1982) via $H 76 \alpha$ observations $\left(V_{L S R}=50.9 \mathrm{~km} \mathrm{~s}^{-1}\right)$ find near $/$ far kinematic distances of $3.9 / 10.5 \mathrm{kpc}$. Using the CS velocity of $V_{\text {LSR }}$ of $54.2 \mathrm{~km} \mathrm{~s}^{-1}$ reported by Bronfman, Nyman, \& May (1996) and the galactic rotation model of Wouterloot et al. (1990), we estimate near and far kinematic distances of 6.5 and $8.0 \mathrm{kpc}$, respectively. Kuchar \& Bania (1990) report HI absorption up to the terminal velocity, and adopt a distance of $8.8 \mathrm{kpc}$. Araya et al. (2002) found a distance of $8.12 \mathrm{kpc}$. Wenger et al. (2018) report a Monte Carlo parallax distance of $7.82 \mathrm{kpc}$. We adopt $8.0 \mathrm{kpc}$ as the distance to G43.89 for our analysis.

The water maser emission toward G43.89 was first detected by Churchwell, Walmsley, \& Cesaroni (1990) and mapped with high spatial resolution by Hofner \& Churchwell (1996). $\mathrm{CH}_{3} \mathrm{OH}$ maser emission at $6.67 \mathrm{GHz}$ was reported by Schutte et al. (1993). Baudry et al. (1997) and Codella et al. (2010) suggest the presence of $\mathrm{OH}$ maser emission. The $\mathrm{NH}_{3}(1,1),(2,2)$ and $(3,3)$ observations of Codella et al. (2010) show weak emission with no distinctive morphology. CS studies were performed by Hatchell et al. (1998) and Olmi \& Cesaroni (1999). Bronfman, Nyman, \& May (1996) detected emission of $\mathrm{CS}(J=2 \rightarrow 1)$. Single-dish $\mathrm{C}_{2} \mathrm{H}_{3} \mathrm{~N}$ and $\mathrm{NH}_{3}$ observations by Olmi, Cesaroni, \& Walmsley (1993) indicate a gas temperature $\mathrm{T} \sim 30 \mathrm{~K}$. The high-excitation $\mathrm{NH}_{3}$ studies of Cesaroni, Walmsley, \& Churchwell (1992) and Olmi, Cesaroni, \& Walmsley (1993) indicate the presence of hot, dense gas, but Hatchell et al. (1998, 2000) find no evidence for a hot molecular core. Single-dish CO observations by Shepherd \& Churchwell (1996) detect a total linewidth of $39.1 \mathrm{~km} \mathrm{~s}^{-1}$ which suggests an outflow. Infrared fluxes were measured and spectra taken by Doherty et al. (1994) and Hanson, Luhman, \& Rieke (2002).

Here, we perform a study of G43.89 similar to that of G12.21 by de la Fuente et al. (2018). Our goals are to investigate where the water masers arise, and to characterize both the ionized and molecular gas components of G43.89. In Section 2 we describe the observations and data reduction procedures. Results and discussion are given in Section 3. Summary and conclusions are given in Section 4 . The physical characterization at $3.6 \mathrm{~cm}$ for UC H II +EE regions of Paper I and the comparison with the Kim \& Koo (2001) study at $20 \mathrm{~cm}$ is shown in Appendix A. The latter helps to confirm G43.89 as an UC H II +EE region.

\section{OBSERVATIONS}

\subsection{Spitzer and low-resolution VLA Observations}

Infrared observations were taken from the Spitzer telescope (Werner et al. 2004) Legacy Programs. IRAC (Fazio et al. 2004) data from the GLIMPSE Spitzer and Ancillary Data (Benjamin et al. 2003) and MIPS (Rieke et al. 2004) $24 \mu \mathrm{m}$ data from MIPSGAL (Carey et al. 2009) were retrieved.

The IR imagery traces extended emission of UC H II regions at arcmin scales. The stellar content and PAHs at $3.3 \mu \mathrm{m}$ are observed in the [3.6] $\mu \mathrm{m}$ IRAC1 band. The ionized gas is detected in the [4.5] $\mu \mathrm{m}$ IRAC2 band. The [5.8] $\mu \mathrm{m}$ IRAC3 band traces cold dust and PAHs at $6.2 \mu \mathrm{m}$. 
Table 1. High Resolution Radio continuum observations

\begin{tabular}{|c|c|c|c|c|c|c|c|}
\hline $\begin{array}{l}\lambda \\
(\mathrm{cm})\end{array}$ & Instrument & $\begin{array}{c}\mathrm{S}_{v}{ }^{\mathrm{b}} \\
(\mathrm{mJy})\end{array}$ & $\begin{array}{c}\text { Beam Size } \\
\left(" x^{\prime \prime}\right)\end{array}$ & $\begin{array}{l}\text { PA } \\
\left({ }^{\circ}\right)\end{array}$ & $\begin{array}{c}\text { Source Size } \\
\left({ }^{c}\right)\end{array}$ & $\begin{array}{c}\mathrm{rms} \\
\left(\mathrm{mJy} \mathrm{beam}^{-1}\right)\end{array}$ & $\begin{array}{c}\text { Observational } \\
\text { Program }\end{array}$ \\
\hline 0.3 & ALMA & 401.0 & $1.90 \times 1.58$ & +57.0 & 2.29 & 0.30 & 2015.1.00280.S \\
\hline 0.3 & OVRO & 260.0 & $3.45 \times 2.45$ & -58.0 & 1.70 & 3.00 & - \\
\hline 0.7 & VLA-CnB & 396.0 & $1.46 \times 0.81$ & +90.0 & 2.00 & 0.80 & AK423 \\
\hline 2.0 & VLA-CnB & 420.0 & $1.58 \times 0.95$ & -08.0 & 2.00 & 0.30 & AK423 \\
\hline 3.6 & VLA-CnB & 570.0 & $2.86 \times 1.85$ & -08.0 & 3.20 & 0.20 & AK423 \\
\hline
\end{tabular}

a Technical details of the low-resolution VLA observations (the $20 \mathrm{~cm}$ NVSS data) are given in Condon et al. (1998)

${ }^{\mathrm{b}}$ Uncertainty on integrated flux is $20 \%$ at 0.3 and $0.7 \mathrm{~cm} ; 10 \%$ at $2 \mathrm{~cm} ; 5 \%$ at $3.6 \mathrm{~cm}$; and $10 \%$ at $20.0 \mathrm{~cm}$.

${ }^{c}$ Deconvolved size obtained using the task imfit of AIPS; $\Theta_{\mathrm{s}}=\sqrt{\Theta_{\mathrm{x}} \Theta_{\mathrm{y}}}$.

Table 2. Parameters of the spectral line observations

\begin{tabular}{lcccccc}
\hline Line & Instrument & $\begin{array}{c}\text { Rest frequency } \\
(\mathrm{GHz})\end{array}$ & $\begin{array}{c}\text { Channel width } \\
\left(\mathrm{km} \mathrm{s}^{-1}\right)\end{array}$ & $\begin{array}{c}\text { rms } \\
\left(\mathrm{mJy} \mathrm{beam}^{-1}\right)\end{array}$ & $\begin{array}{c}\text { Beam Size } \\
\left({ }^{\prime \prime} \times{ }^{\prime \prime}\right)\end{array}$ & $\begin{array}{c}\text { PA } \\
\left({ }^{\circ}\right)\end{array}$ \\
\hline $\mathrm{H} 40 \boldsymbol{\alpha}$ & ALMA & 99.02296 & 3.0 & 1.5 & $1.68 \times 1.34$ & +66 \\
$\mathrm{H} 41 \boldsymbol{\alpha}$ & OVRO & 92.03444 & 3.3 & 25.0 & $3.45 \times 2.45$ & -58 \\
$\mathrm{H} 42 \boldsymbol{\alpha}$ & ALMA & 85.68839 & 3.0 & 2.0 & $1.92 \times 1.68$ & +64 \\
${ }^{13} \mathrm{CS}(\boldsymbol{J}=2 \rightarrow 1)$ & OVRO & 92.49431 & 1.6 & 35.0 & $3.45 \times 2.45$ & -58 \\
$\mathrm{CS}(\boldsymbol{J}=2 \rightarrow 1)$ & ALMA & 97.98095 & 3.0 & 2.0 & $1.67 \times 1.43$ & +68 \\
$\mathrm{HCN}(\boldsymbol{J}=1 \rightarrow 0)$ & ALMA & 88.63160 & 3.0 & 2.0 & $1.86 \times 1.63$ & +59 \\
$\mathrm{H}^{13} \mathrm{CN}(\boldsymbol{J}=1 \rightarrow 0)$ & ALMA & 86.33992 & 3.0 & 2.0 & $1.91 \times 1.65$ & +62 \\
$\mathrm{HCO}(\boldsymbol{J}=1 \rightarrow 0)$ & ALMA & 89.18852 & 3.0 & 2.0 & $1.85 \times 1.63$ & +60 \\
$\mathrm{SiO}(\boldsymbol{J}=2 \rightarrow 1)$ & ALMA & 86.84696 & 3.0 & 2.0 & $1.89 \times 1.65$ & +62 \\
\hline
\end{tabular}

The $[8.0] \mu \mathrm{m}$ IRAC4 band traces PAHs where the $7.7 \mu \mathrm{m}$ emission is predominant. The MIPS [24] $\mu \mathrm{m}$ band detects warm dust and embedded YSOs as point-like sources. More details and a description of characteristics in these bands in UC H II +EE regions is presented in Paper I (and references therein) (see also Robitaille et al. 2006, 2008; de la Fuente et al. 2009a,b).

Because there are no VLA observations at $3.6 \mathrm{~cm}$ in configurations C or D for G43.89, we follow Kurtz et al. (1999) and use $20 \mathrm{~cm}$ NVSS observations from Condon et al. (1998) to trace the EE. NVSS has a sensitivity to structures $7^{\prime}-15^{\prime}$, and the EE seems to be associated with IR Spitzer bubbles (see Paper I), with the NVSS emission encompassing the arcminute-scale IR structures.

\subsection{High resolution observations}

\subsubsection{VLA and OVRO observations}

VLA observations of RC at $0.7,2$, and $3.6 \mathrm{~cm}$ toward G43.89 were performed simultaneously with those of G12.21, reported by de la Fuente et al. (2018). In a similar way to G12.21, we used the Owens Valley Radio Observatory (OVRO) Millimeter Array to observe RC emission at 0.3 $\mathrm{cm}$, and the ${ }^{13} \mathrm{CS}(J=2 \rightarrow 1)\left(v_{0}=92.49430 \mathrm{GHz}\right)$ line and the $\mathrm{H} 41 \alpha\left(v_{0}=92.03445 \mathrm{GHz}\right)$ line emission toward G43.89. The observations were carried out in March and April, 1996, using both equatorial and high-resolution configurations. Details of these observations, instrumental set-up, calibration and data reduction procedures are presented in de la Fuente et al. (2018).
Calibration and standard data reduction procedures were performed using the AIPS package ${ }^{1}$. Observational parameters for the high-resolution $\mathrm{RC}$ are shown in Table 1. Table 2 gives the corresponding parameters for the spectral line observations.

\subsubsection{ALMA observations}

We retrieved archival Atacama Large Millimetre Array (ALMA) data of the project 2015.1.00280.S (PI: R. Cesaroni) to study the molecular, mm-continuum, and RRL emission associated with G43.89. The observations were performed using Band $3(84-116 \mathrm{GHz})$ receivers and include two Executions Blocks (EBs) obtained on the 16th and 17th of March, 2016, with 37 and 40 12-m antennas, respectively.

A total of six spectral windows with different bandwidths and spectral resolutions were used to target the continuum and line emission.

The highest and lowest spectral resolution of the observations are $0.42 \mathrm{~km} \mathrm{~s}^{-1}$ and $1.48 \mathrm{~km} \mathrm{~s}^{-1}$, respectively. The total observation time on G43.89 was 35 minutes (including both EBs). The baselines of the array covered a range from $15.1 \mathrm{~m}$ to $460.0 \mathrm{~m}$, which provided an angular resolution of $\sim 1{ }^{\prime \prime} 5$ and a maximum recoverable scale of $\sim 25^{\prime \prime}$.

Precipitable water vapour levels ranged from 2.3 to

1 AIPS is produced and maintained by the National Radio Astronomy Observatory, a facility of the National Science Foundation operated under cooperative agreement by Associated Universities, Inc. 
$2.9 \mathrm{~mm}$ and the typical system temperature was 50 $100 \mathrm{~K}$ for different antennas. The data were calibrated with the ALMA pipeline (36252-Pipeline-Cycle3-R4-B; CASA version 4.5.2 $\mathrm{r} 36115)$ using $\mathrm{J} 1751+0939(\sim 2.72 \mathrm{Jy}$ at $86.751 \mathrm{GHz}$ ) as a flux/bandpass calibrator and J1922+1530 $\sim 260 \mathrm{mJy}$ as a gain calibrator.

Images were created using Briggs weighting with the robust parameter set to -0.5 in order to improve the spatial resolution, at the cost of slightly higher noise. The $0.3 \mathrm{~cm}$ continuum image has an average rms of $0.3 \mathrm{mJy} \mathrm{beam}^{-1}$. The corresponding molecular line observational parameters are summarized in Table 2.

\section{RESULTS AND DISCUSSION}

We study the EE associated with G43.89 via Spitzer and VLA low-resolution observations. The need for this study was evident upon visual inspection of the MIPS image, which shows significant structure around the saturated UC H II region .

\subsection{The extended emission}

IRAC and IRAC+MIPS RGB images for G43.89 are shown in Fig. 1. The IRAC morphology is similar to other UC H II +EE regions shown by de la Fuente et al. (2009a,b) with the EE marked by the red emission at $8.0 \mu \mathrm{m}$. In G43.89, the emission at $4.5 \mu \mathrm{m}$ is considerable and matches the south-western edge of the $8 \mu \mathrm{m}$ morphology (in red), being more intense at the position of the UC H II region (in yellow) marked with a square in Fig. 1 (left). An almost circular structure with size $\sim 1^{\prime}$, considered as a cavity, is seen at $8 \mu \mathrm{m}$ and delineates a bubble-like shape that is characteristic of some star forming regions in the Milky Way (Churchwell et al. 2006) as revealed by Spitzer observations.

The IRAC+MIPS image shows the same behaviour observed in UC H II +EE regions from Paper I, showing a remarkable similarity with G37.55-0.11. In both sources, lowresolution $\mathrm{RC}$ contours coincide with the $24 \mu \mathrm{m}$ emission; a well-traced cavity is evident at $8 \mu \mathrm{m}$, which is filled with $24 \mu \mathrm{m}$ emission, and red, point-like sources, YSOs or dust condensations, are located inside. Red contours, coinciding with the saturation at $24 \mu \mathrm{m}$ (cyan color), are from the high-resolution VLA $3.6 \mathrm{~cm}$ image, also shown in Fig. 2. This emission traces the UC component.

The NVSS emission has two peaks, one coinciding with the UC H II region position (peak-1), the other coinciding with the IRAC cavity (peak-2). Weaker RC emission extends to the $\mathrm{NE}$ as part of a larger structure of ionized gas. It is noticeable that at larger scales, the RC emission matches with $24 \mu \mathrm{m}$ emission but does not have an IRAC counterpart (such behaviour is also seen in G37.55-0.11). The IRAC emission at $4.5 \mu \mathrm{m}$ in peak-1 matches with the yellow emission on the IRAC+MIPS image, supporting the presence of ionized gas at several scales from the UC H II regions.

Following Kurtz et al. (1999), we see that for both the extended and the compact emission (the former traced by NVSS, the latter by the $0.7,2$ and $3.6 \mathrm{~cm}$ images), there is continuity at the peak-1 position (see Fig. 1 and 2). Moreover, a result from Paper I is that $24 \mu \mathrm{m}$ emission coin- cides with the RC emission at $3.6 \mathrm{~cm}$ at several scales - including the saturated UC component. This MIPS emission could indicate the presence of ionized gas at intermediate scales between the VLA high-resolution and low-resolution observations. VLA observations at $3.6 \mathrm{~cm}$ in the $\mathrm{C}$ or Dconfiguration could confirm this. MIPS emission at peak-1 is between $20^{\prime \prime}$ (the saturated zone) and $70^{\prime \prime}$ (including first Airy ring), in agreement with the size of $45^{\prime \prime}$ (1.75 pc) obtained from the NVSS image. The physical parameters of the EE and the UC H II are determined in the standard way (equations A1 to A4 in Appendix A), and these results are shown in Table 3.

These physical parameters agree with those reported in Table A1 for sources in Paper I and Kim \& Koo (2001) as shown in Appendix A. Considering the calculated values and the morphological study described above, G43.89-0.78 can be catalogued as an UC H II +EE . However, intermediate resolution VLA observations at $3.6 \mathrm{~cm}$, such as those presented in Paper I, would be useful to strengthen this result.

García-Segura \& Franco (1996) show that wind driven bubbles formed by the expansion of the UC H II regions produce structures that are not larger than a scale of $\sim 2 \mathrm{pc}$. From Fig. 1 it is clear that the RC emission of the UC H II in G43.89 extends beyond $\sim 4$ pc. Therefore, it is likely that the expansion of a bubble driven by a wind cannot be applied to explain all of the Spitzer bubbling structures observed nearby G43.89.

On the other hand, star forming regions with molecular nebulosity from the parent cloud(s) have been modelled as the result of the collision of two molecular clouds triggering massive star formation; i.e., the cloud-cloud collision (CCC) mechanism (Nagazawa \& Miyama 1987; Habe \& Ohta 1992). This model has been applied to giant molecular clouds turning into rich clusters of high-mass stars (e.g., Furukawa, Dawson \& Ohama 2009; Ohama, Dawson \& Furukawa 2010; Fukui et al. 2014; Baug et al. 2016; Kuwahara et al. 2020), and to smaller molecular clouds that trigger the formation of clusters with one or a few (OB type) massive stars (e.g., Torii et al. 2011, 2015, 2017; Fujita et al. 2019). With high resolution CO velocity maps it would be viable to model emission at large scales ( $\gtrsim 2 \mathrm{pc}$ ) around UC H II +EE regions with the CCC scenario.

In several cases in Paper I we note the presence of molecular nebulosity, with the embedded UC component resembling the clump at a shocked interface between colliding clouds. The extended emission also resembles the bubble structures left behind where one or more newly-formed stars ionize a cavity inside the larger cloud (Spitzer bubbles). Such a study could confirm if the RC morphological classification of UC H II +EE regions is related with the clouds' initial conditions, such as mass, velocity dispersion, velocity directions and viewing angle (see Torii et al. 2015, for their "cup" and "tunnel" orientation discussion). In particular, for the case of G43.89, this model could explain the presence of peaks -1 and 2 in the same region.

\subsection{The Ultracompact vicinity}

\subsubsection{RC and RRL emission}

The high-resolution $\mathrm{RC}$ maps at $0.7,2$, and $3.6 \mathrm{~cm}$, tracing the UC component, are presented in Fig. 2. These maps 

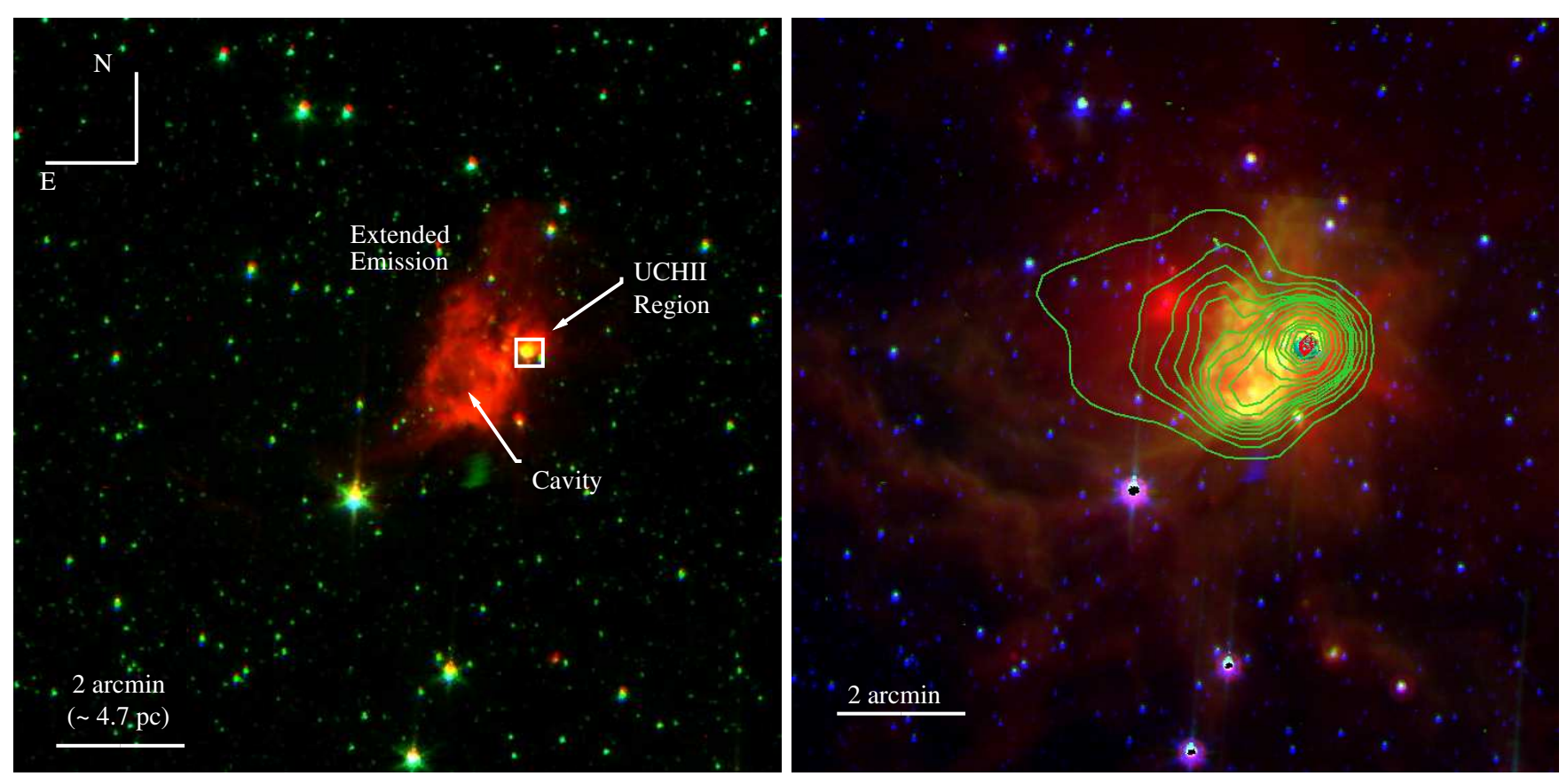

Figure 1. G43.89-0.78. Left: Spitzer IRAC RGB $(8 \mu \mathrm{m}+4.5 \mu \mathrm{m}+3.6 \mu \mathrm{m})$ image. The red emission traces the extended emission. The square shows the position of the UC H II region. A cavity-like structure is marked. The yellow emission at the position of the UC H II region is from the intense $4.5 \mu \mathrm{m}$ emission. This emission is clearly observed in its corresponding IRAC image, and matches very well with the red emission. Right: Spitzer IRAC+MIPS RGB $(24 \mu \mathrm{m}+8 \mu \mathrm{m}+4.5 \mu \mathrm{m})$ image. Green contours are from NVSS showing the $20 \mathrm{~cm}$ emission tracing extended ionized gas. The levels are $-4,4,8,16,32 \ldots$ times $1.20 \mathrm{mJy} \mathrm{beam}^{-1}$. The NVSS peak emission, at the position of the UC H II region, is coincident with the emission at $3.6 \mathrm{~cm}$ (red contours). In this small region, also $24 \mu \mathrm{m}$ emission is saturated (cyan colour). The emission at $4.5 \mu \mathrm{m}$ and $8 \mu \mathrm{m}$, shown here in yellow, matches with higher values of NVSS contours. The NVSS emission has two peaks, one related with the UC component (peak-1), and other related with the cavity (peak-2). At a distance of $8 \mathrm{kpc}, 2^{\prime}$ corresponds to $\sim 4.7 \mathrm{pc}$.

Table 3. Results for the ionized gas

\begin{tabular}{lcccccccccc}
\hline $\begin{array}{l}\text { H II } \\
\text { Component }\end{array}$ & $\begin{array}{c}\mathrm{S}_{v} \\
(\mathrm{mJy})\end{array}$ & $\begin{array}{c}\text { Distance } \\
(\mathrm{kpc})\end{array}$ & $\begin{array}{c}\text { Source } \\
\text { size }\left({ }^{\prime \prime}\right)\end{array}$ & $\begin{array}{c}\text { Source } \\
\text { size }(\mathrm{pc})\end{array}$ & $\begin{array}{c}\mathrm{T}_{\mathrm{e}}^{\mathrm{a}} \\
(\mathrm{K})\end{array}$ & $\begin{array}{c}\text { EM } \\
\left(\mathrm{cm}^{-6} \mathrm{pc}\right)\end{array}$ & $\begin{array}{c}\mathrm{n}_{\mathrm{e}} \\
\left(\mathrm{cm}^{-3}\right)\end{array}$ & $\begin{array}{c}\mathrm{M}_{\mathrm{H} \text { II }} \\
\mathrm{M}_{\odot}\end{array}$ & $\begin{array}{c}\text { Log }\left(\mathrm{N}_{\mathrm{c}}^{\prime}\right) \\
\left(\mathrm{s}^{-1}\right)\end{array}$ & $\begin{array}{c}\text { Spectral } \\
\text { Type }^{\mathrm{b}}(\mathrm{radio})\end{array}$ \\
\hline Extended Emission & $318^{\mathrm{c}}$ & 8.0 & 45.0 & 1.75 & 10000 & $8.7 \times 10^{4}$ & $2.2 \times 10^{2}$ & 16.00 & 48.10 & $\mathrm{O} 9$ \\
Ultracompact & $570^{\mathrm{d}}$ & 8.0 & 3.2 & 0.12 & 10700 & $3.7 \times 10^{7}$ & $1.8 \times 10^{4}$ & 0.43 & 48.44 & $\mathrm{O} 8$ \\
\hline
\end{tabular}

${ }^{a}$ We adopt the canonical value of $1 \times 10^{4} \mathrm{~K}$ for the $\mathrm{EE}$, and for the $\mathrm{UC}$ we use the electron temperature derived from the $\mathrm{H} 41 \alpha$ emission using equation 1 with values from table 2).

b Using Panagia (1973). All sources considered at ZAMS.

c This flux corresponds to the NVSS peak- 1 only.

$\mathrm{d}$ This flux corresponds to the VLA CnB conf. at $3.6 \mathrm{~cm}$

Table 4. Results of the RRL observations

clearly show the cometary morphology of the UC H II region. The positions of the two water maser features reported

\begin{tabular}{cccccc}
\hline Line & $\begin{array}{c}\mathrm{S}_{v}{ }^{\mathrm{a}} \\
(\mathrm{mJy})\end{array}$ & $\begin{array}{c}\mathrm{Siz}^{\mathrm{b}} \\
\left({ }^{\prime}\right)\end{array}$ & $\begin{array}{c}\mathrm{V}_{\mathrm{LSR}} \\
\left(\mathrm{km} \mathrm{s}^{-1}\right)\end{array}$ & $\begin{array}{c}\Delta \mathrm{V}(\mathrm{FWHM}) \\
\left(\mathrm{km} \mathrm{s}^{-1}\right)\end{array}$ & $\begin{array}{c}\mathrm{T}_{\mathrm{L}} / \mathrm{T}_{\mathrm{C}} \text { by Hofner \& Churchwell (1996) are indicated with crosses on } \\
(\text { at } 0.3 \mathrm{~cm}) \text { the images. The water masers are not spatially coincident }\end{array}$ \\
\hline $\mathrm{H} 40 \alpha$ & 386.0 & 2.2 & $55.2 \pm 0.1$ & $24.4 \pm 0.2$ & $0.95 \pm 0.01$ with the peak of RC observed with the VLA, but rather are \\
$\mathrm{H} 41 \alpha$ & 246.0 & 3.0 & $56.6 \pm 0.4$ & $25.8 \pm 0.9$ & $0.95 \pm 0.02$ offset from the head of the cometary structure. Thus, the wa- \\
$\mathrm{H} 42 \alpha$ & 378.0 & 2.4 & $55.3 \pm 0.1$ & $24.3 \pm 0.3$ & $0.95 \pm 0.01$ ter masers are not coincident with the ionized gas. The fact \\
that there is 3.6 cm RC emission partially overlapping with
\end{tabular}

a Uncertainties on integrated flux are 20\%. Typical OVRO beam sizethe water masers is due to the limited angular resolution for the $0.3 \mathrm{~cm}$ lines are $3^{\prime \prime}$. Uncertainties on integrated flux for ALMaf these observations. Indeed, convolving our higher angular observations are $5 \%$. resolution maps at 2 and $0.7 \mathrm{~cm}$ with a larger beam repro-

${ }^{\mathrm{b}}$ Deconvolved size obtained from observations using task imfit of AIPduces this effect. In addition, Hofner \& Churchwell (1996) $\Theta_{\mathrm{s}}=\sqrt{\Theta_{\mathrm{x}} \Theta_{\mathrm{y}}}$ mention that they found the masers to be "well-separated" 


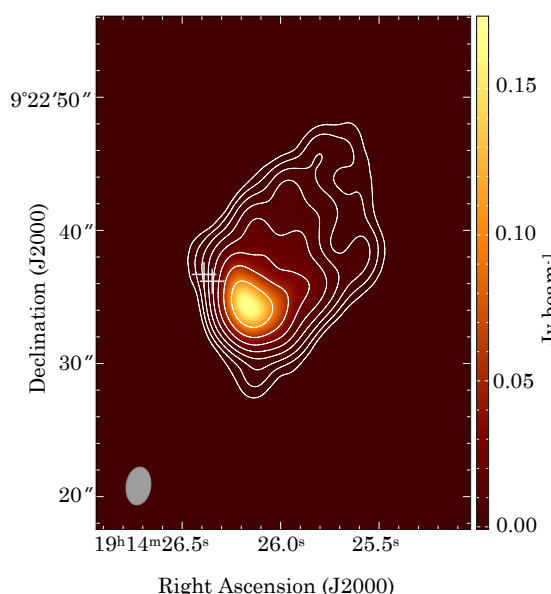

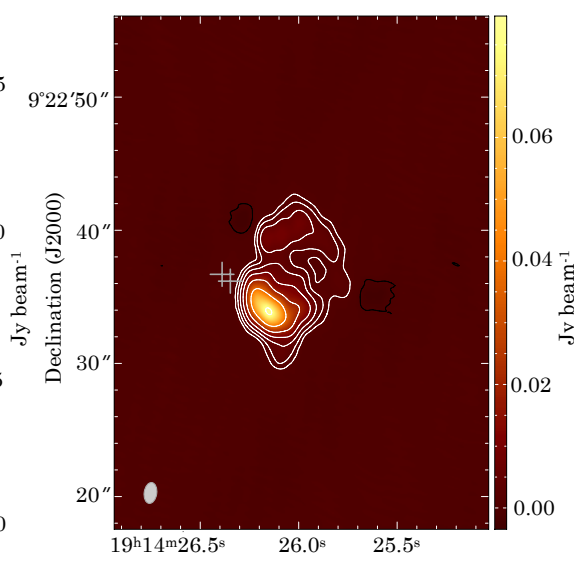

Right Ascension (J2000)

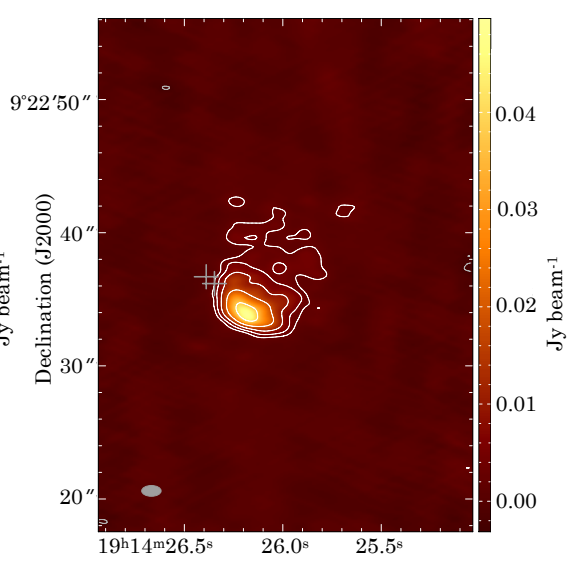

Right Ascension (J2000)

Figure 2. Radio continuum emission for G43.89-0.78 at 3.6, 2, and $0.7 \mathrm{~cm}$ observed with the VLA at high-resolution. The contours increase as $\pm 4 \times \mathrm{rms} \times 2^{i}$ with $i=0,1,2,3 \ldots$ (black contours have negative values) and the rms is listed in Table 1 . The synthesized beams (see Table 1) are shown in the bottom-left corner of each panel. The crosses mark the position of the water masers from Hofner \& Churchwell (1996). These maps trace only the UC component.
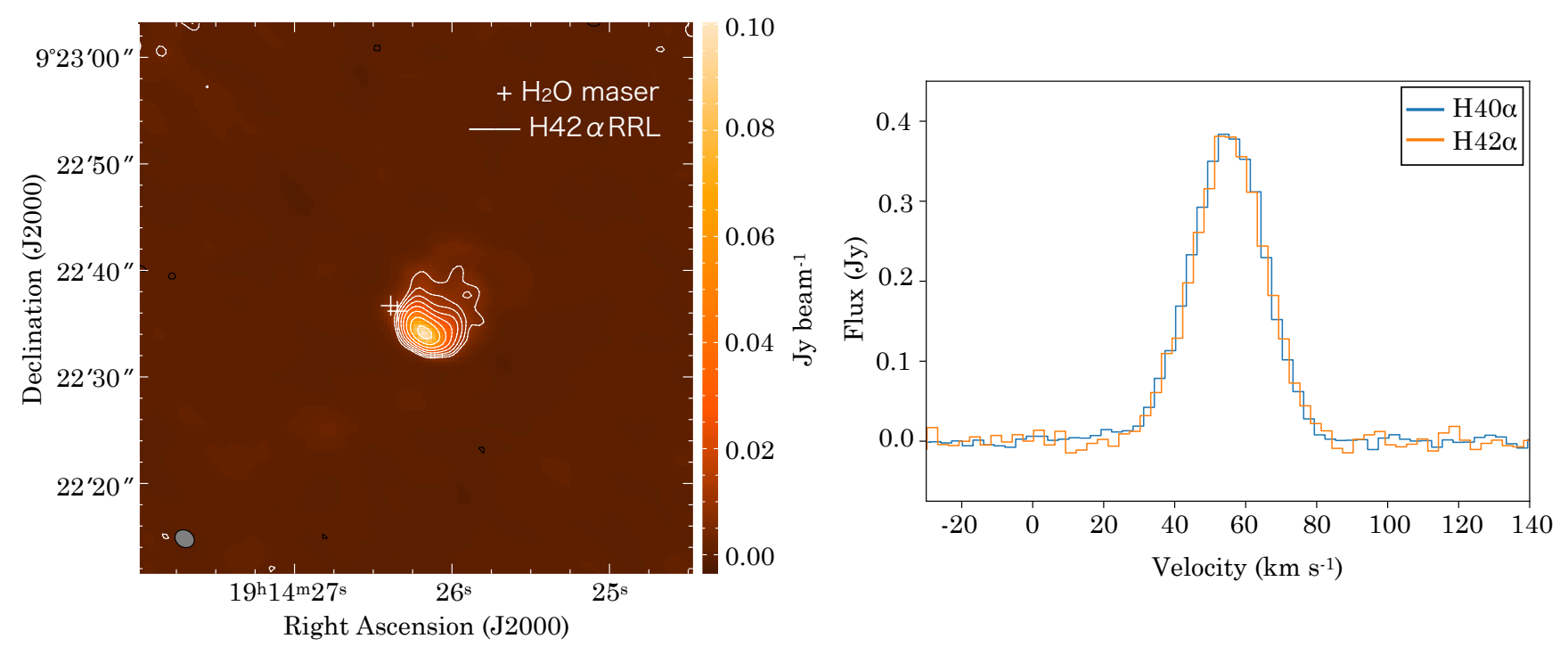

Figure 3. Continuum at $v \sim 92.856 \mathrm{GHz}$ and RRL emission of G43.89-0.78 obtained from ALMA observations. Left: The colour map corresponds to the continuum emission at $0.3 \mathrm{~cm}$ and the contours represent the velocity-integrated emission of the H42 $\alpha$ line obtained by integrating emission in the velocity range from $\sim 0$ to $100 \mathrm{~km} \mathrm{~s}^{-1}$, ensuring that all the line is included. The contours increase as $\pm 5 \times \mathrm{rms} \times 1.5^{i}$ with $i=0,1,2,3 \ldots$ (black contours have negative values) and the rms is listed on Table 2 . The synthesized beam (see Table 2) is shown in the bottom-left corner. The crosses mark the position of the water masers from Hofner \& Churchwell (1996). Right: Continuum subtracted line profiles of the $\mathrm{H} 40 \alpha$ and $\mathrm{H} 42 \alpha$ emission.

Table 5. Physical parameters (continuum) for clumps on G43.89 vicinity

\begin{tabular}{|c|c|c|c|c|c|c|c|c|}
\hline $\begin{array}{l}\text { Clump }^{a} \\
\text { Designation }\end{array}$ & $\begin{array}{c}\text { Otherb } \\
\text { Designation }\end{array}$ & $\begin{array}{l}\text { Right Ascension } \\
(\mathrm{J} 2000: \mathrm{h} \mathrm{m} \mathrm{s})\end{array}$ & $\begin{array}{l}\text { Declination } \\
(\mathrm{J} 2000: \circ, \prime \prime)\end{array}$ & $\begin{array}{c}\mathrm{S}_{v}{ }^{\mathrm{c}} \\
(\mathrm{mJy})\end{array}$ & $\begin{array}{l}\text { Sourced } \\
\text { size (") }\end{array}$ & $\begin{array}{l}\mathrm{T}_{\text {dust }} \\
(\mathrm{K})\end{array}$ & $\begin{array}{l}\mathrm{M}_{\mathrm{H}_{2}} \\
\left(\mathrm{M}_{\odot}\right)\end{array}$ & $\begin{array}{c}24 \mu \mathrm{m} \\
\text { Counterpart }\end{array}$ \\
\hline G43.899-0.786 & G43 N & 191427.818 & +092300.33 & 6.00 & 1.34 & 50.00 & 11.00 & $\mathrm{Y}$ \\
\hline G43.888-0.787 & G43 S & 191426.789 & +092225.12 & 8.00 & 2.60 & 50.00 & 15.00 & $\mathrm{~N}$ \\
\hline
\end{tabular}

\footnotetext{
${ }^{\text {a }}$ Based on galactic coordinates.

b Respect to the main clump: G43(C) or G43.890-0.784,

${ }^{\mathrm{c}}$ Integrated flux. Uncertainties of $5 \%$.

${ }^{\mathrm{d}}$ Deconvolved size obtained from observations using CASA. $\Theta_{\mathrm{s}}=\sqrt{\Theta_{\mathrm{x}} \Theta_{\mathrm{y}}}$
} 

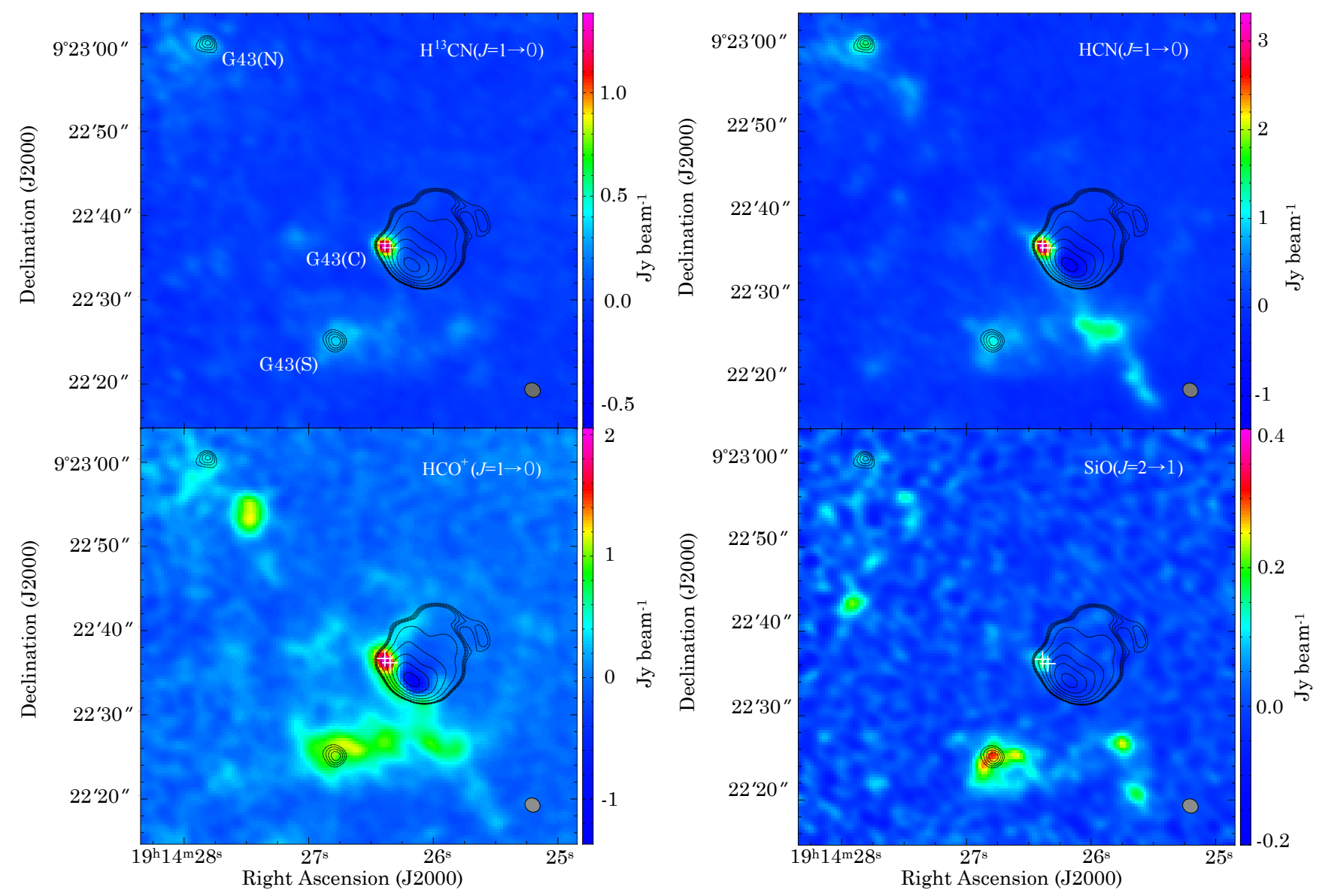

Figure 4. ALMA observations of the continuum emission as well as line emission of different molecular species in the vicinity of G43.89-0.78. The black contours represent the continuum emission at $0.3 \mathrm{~cm}$. The first four contours are $5,6,7$ and 8 times the rms of the map (listed in Table 2) and then they increase as $\pm 8 \times \mathrm{rms} \times 2^{i}$ with $i=0,1,2,3 \ldots$. Apart from the cometary UC HII region, three other continuum clumps were identified and they are indicated in the upper-left panel as G43(N), G43(C) and G43(S) (see main text). The water masers from Hofner \& Churchwell (1996) are marked as white crosses.

from the cometary ionized arc, which is a result of their relatively high angular resolution $\left(\sim 0 !^{\prime \prime} 5\right)$ observations.

Fig. 3 shows the $0.3 \mathrm{~cm}$ continuum, $\mathrm{H} 40 \alpha$, and $\mathrm{H} 42 \alpha$ RRL emission obtained from ALMA observations. A contour map of the velocity-integrated H $42 \alpha$ RRL emission, superimposed on a colour map of the continuum emission, is shown in the left panel, while the profiles of the $\mathrm{H} 40 \alpha$ and $H 42 \alpha$ lines are shown in the right panel. The velocityintegrated map of the H $42 \alpha$ RRL was obtained by integrating emission in the velocity range from $\sim 0$ to $100 \mathrm{~km} \mathrm{~s}^{-1}$. A Gaussian fit to the profiles of the H $40 \alpha$ and H42 $\alpha$ RRLs gives a peak velocity $\mathrm{V}_{\mathrm{LSR}}=55.2 \pm 0.1 \mathrm{~km} \mathrm{~s}^{-1}$ and a FWHM $\Delta \mathrm{V}=24.3 \pm 0.3 \mathrm{~km} \mathrm{~s}^{-1}$, which is in agreement with the systemic velocity $\mathrm{V} \sim 55 \mathrm{~km} \mathrm{~s}^{-1}$ obtained from previous molecular observations (Olmi, Cesaroni, \& Walmsley 1993; Bronfman, Nyman, \& May 1996; Olmi \& Cesaroni 1999; Araya et al. 2002). A fit to the H41 $\alpha$ line observed with OVRO gives a similar result (see Table 4). These results indicate that the ionized gas and the molecular gas are physically associated.

From the ratio of the continuum and RRL fluxes, and the line-width of the RRL, it is possible to determine the LTE electronic temperature, $\mathrm{T}_{\mathrm{e}}$, of the ionized gas as (Mezger \& Henderson 1967; Dupree \& Goldberg 1970):

$$
\left(\frac{\mathrm{T}_{\mathrm{e}}}{\mathrm{K}}\right)=2.2 \times 10^{3}\left(\frac{v}{\mathrm{GHz}}\right)^{0.96}\left(\frac{\mathrm{T}_{\mathrm{L}}}{\mathrm{T}_{\mathrm{C}}}\right)^{-0.87}\left(\frac{\Delta \mathrm{V}}{\mathrm{km} \mathrm{s}^{-1}}\right)^{-0.87},
$$

where $v$ is the observation frequency, $\mathrm{T}_{\mathrm{C}}$ is the brightness temperature of the continuum emission, and $\mathrm{T}_{\mathrm{L}}$ and $\Delta \mathrm{V}$ are the continuum-subtracted peak brightness temperature and the line-width of the RRL, respectively. Since $\mathrm{S}_{\mathrm{L}} / \mathrm{S}_{\mathrm{C}}$ is $\sim 0.95$ (see Table 4 ), equation 1 gives an LTE $\mathrm{T}_{\mathrm{e}} \sim 1.1 \times 10^{4} \mathrm{~K}$. The physical parameters obtained for the UC H II region in G43.89, using this temperature value, are presented in Table 3 . For the calculations we used the $3.6 \mathrm{~cm}$ data and a deconvolved size of the source $\simeq 3^{\prime \prime}(\simeq 0.12 \mathrm{pc})$. The derived values of the physical parameters for this source are in approximate agreement with those obtained by Wood \& Churchwell (1989). 
Table 6. Results of the molecular line observations of G43(C) or G43.890-0.784.

\begin{tabular}{lccrccc}
\hline Line & $\begin{array}{c}\text { Peak }^{\mathrm{a}} \\
(\mathrm{Jy})\end{array}$ & $\begin{array}{c}\mathrm{V}_{\mathrm{LSR}} \\
\left(\mathrm{km} \mathrm{s}^{-1}\right)\end{array}$ & $\begin{array}{r}\Delta \mathrm{V}(\mathrm{FWHM}) \\
\left(\mathrm{km} \mathrm{s}^{-1}\right)\end{array}$ & $\begin{array}{c}\text { Column Density } \\
\left(\mathrm{cm}^{-2}\right)\end{array}$ & $\begin{array}{c}\text { Abundance } \\
(\mathrm{K})\end{array}$ \\
\hline${ }^{13} \mathrm{CS}(\boldsymbol{J}=2 \rightarrow 1)$ & $<0.035$ & $\ldots$ & $\ldots$ & $\ldots$ & $\ldots$ & $\ldots$ \\
$\mathrm{CS}(\boldsymbol{J}=2 \rightarrow 1)$ & 1.05 & $55.3 \pm 0.1$ & 4.0 & $1.00 \times 10^{16}$ & $5 \times 10^{-9}$ & 18 \\
$\mathrm{HCN}(\boldsymbol{J}=1 \rightarrow 0)$ & 0.68 & $55.3 \pm 0.1$ & 5.0 & $1.00 \times 10^{16}$ & $5 \times 10^{-9}$ & 16 \\
$\mathrm{H}^{13} \mathrm{CN}(\boldsymbol{J}=1 \rightarrow 0)$ & 0.27 & $55.3 \pm 0.1$ & 10.0 & $1.67 \times 10^{14}$ & $8 \times 10^{-11}$ & 16 \\
$\mathrm{HCO}(\boldsymbol{J}=1 \rightarrow 0)$ & 1.60 & $55.3 \pm 0.1$ & 3.0 & $4.00 \times 10^{15}$ & $2 \times 10^{-9}$ & 35 \\
$\mathrm{SiO}(\boldsymbol{J}=2 \rightarrow 1)$ & 0.025 & $55.3 \pm 0.1$ & 15.0 & $1.60 \times 10^{13}$ & $8 \times 10^{-12}$ & 18 \\
\hline
\end{tabular}

${ }^{\text {a }}$ Uncertainties on integrated flux are $20 \%$. Typical OVRO beam sizes for the $0.3 \mathrm{~cm}$ lines are 3 ". Uncertainties on integrated flux for ALMA observations are 5\%.

${ }^{\mathrm{b}}$ Deconvolved size obtained from observations using task imfit of AIPS. $\Theta_{\mathrm{s}}=\sqrt{\Theta_{\mathrm{x}} \Theta_{\mathrm{y}}}$

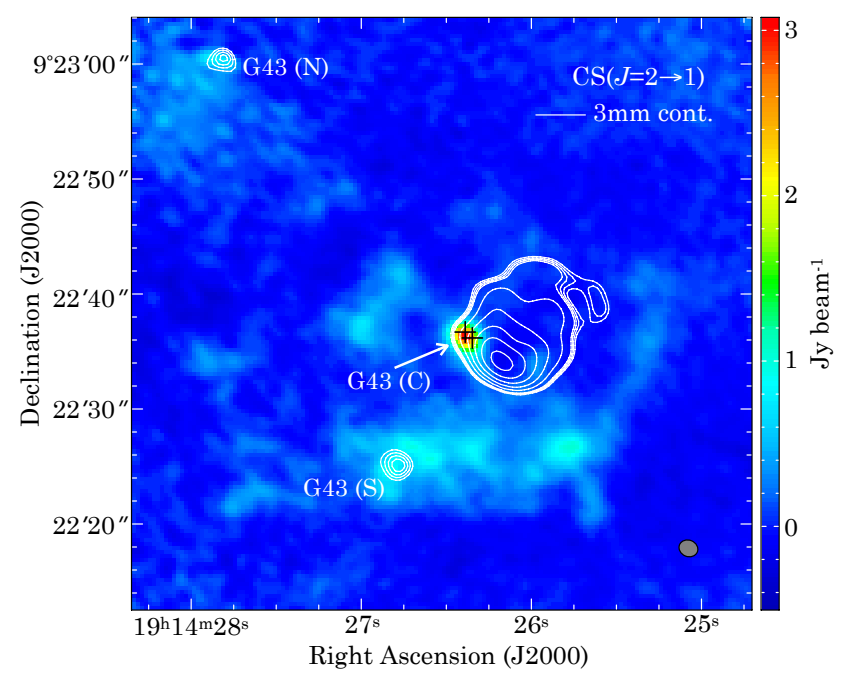

Figure 5. Continuum emission in G43.89-0.78 obtained from the ALMA observations. The colour map corresponds to the velocity-integrated $\operatorname{CS}(J=2 \rightarrow 1)$ emission integrated over the velocity range $15<\mathrm{V}\left(\mathrm{km} \mathrm{s}^{-1}\right)<95$. The $\mathrm{rms}$ of the image is $8.5 \times 10^{-2} \mathrm{Jy} \mathrm{beam}^{-1} \cdot \mathrm{km} \mathrm{s}^{-1}$. The contours represent the continuum emission at $v \sim 92.856 \mathrm{GHz}$. The first four contours are 5 , 6,7 and 8 times the rms of the map (listed on Table 2) and then they increase as $8 \times \mathrm{rms} \times 2^{i}$ with $i=0,1,2,3 \ldots$. The ellipse at the bottom-right corner represents the size of the synthesized beam given in Table 2. The positions of the water masers reported by Hofner \& Churchwell (1996) are indicated with black crosses and coincide with the molecular clump labelled as G43(C). Two extra radio-continuum clumps labelled as $\mathrm{G} 43(\mathrm{~N})$ and $\mathrm{G} 43(\mathrm{~S})$ are observed in the field.

\subsubsection{ALMA and OVRO: Molecular and mm-continuum emission}

Using the JCMT, Hatchell et al. (1998) searched for molecular emission toward 14 UC H II regions including G43.89. For G43.89 they detected several molecular lines $\left(\mathrm{C}^{17} \mathrm{O}\right.$, $\mathrm{C}^{18} \mathrm{O}$, SO, $\left.\mathrm{C}^{34} \mathrm{~S}\right)$, and weak emission from the lowest excitation energy lines of $\mathrm{CH}_{3} \mathrm{CCH}$ and $\mathrm{CH}_{3} \mathrm{OH}$. Also, Li et al. (2015) searched for sulphur-bearing molecules, including OCS, CS, $\mathrm{H}_{2} \mathrm{~S}$ and SO; they only detected emission from $\mathrm{SO}\left(5_{6}-4_{5}\right)$.

From the ALMA observations, emission from $\mathrm{CS}(J=2 \rightarrow 1), \quad \operatorname{HCN}(J=1 \rightarrow 0), \quad \mathrm{H}^{13} \mathrm{CN}(J=1 \rightarrow 0)$,
$\operatorname{HCO}^{+}(J=1 \rightarrow 0), \quad \mathrm{SiO}(J=2 \rightarrow 1) \quad$ lines were detected (see Figs. 4 and 6). On the other hand, no OVRO ${ }^{13} \mathrm{CS}(J=2 \rightarrow 1)$ line emission was detected above a level of $3 \sigma\left(\sigma=0.35 \mathrm{mJy}\right.$ beam $\left.^{-1}\right)$ toward the UC H II region. This is almost certainly due to the limited sensitivity of OVRO observations with respect to ALMA.

Aside from the UC H II region, two more radiocontinuum clumps were detected in the field of view of the ALMA observations. They are marked as G43(N) and G43(S) in Figs. 4 and 5. By their galactic coordinates, these sources are designated as G43.899-0.786 and G43.888-0.787, respectively. $\mathrm{G} 43(\mathrm{~N})$ is coincident with a $24 \mu \mathrm{m}$ point-source within the positional uncertainties. This clump could be a proto-stellar source. On the other hand, G43(S) does not have a $24 \mu \mathrm{m}$ counterpart, although there is considerable molecular emission at its position. G43(N), in this regard, shows only weak molecular emission. Physical parameters for these clumps are reported in Table 5 .

In addition, close inspection of the continuum emission associated with the UC H II region reveals that there is a bump toward the position of the water masers, which also coincides with a bright molecular clump. We label this source as G43(C) with galactic designation G43.890-0.784. Figs. 4 and 5 shows that except for the $\operatorname{SiO}(J=2 \rightarrow 1)$ emission, the molecular emission peaks in $\mathrm{G} 43(\mathrm{C})$. In these figures, it is clear that the water masers are associated with G43(C). The coordinates of this brightest clump are R.A.(J2000) $=19 \mathrm{~h}$ $14 \mathrm{~m} \mathrm{26.387s,} \mathrm{Dec.(J2000)}=9^{\circ} 22^{\prime} 36^{\prime \prime} 28$. The configuration of the cometary UC H II region with a molecular clump and water masers resembles that of G12.21, where de la Fuente et al. (2018) concluded it is a hot molecular core. Thus, in order to assess whether G43.89 also contains a hot molecular core we proceed to study the molecular clump of G43.89 in a similar way as for G12.21.

To determine the physical parameters of the molecular gas in G43(C) we used the CASSIS software, the CDMS spectroscopic databases, the LAMDA molecular databases, and the RADEX code ${ }^{2}$. We extracted spectra from all the molecular species detected with ALMA and then we fitted a model by varying parameters such as the kinetic temperature, and column densities. Fig. 6 shows the fits to the observations. The best fit gives a molecular hydrogen col-

2 CASSIS has been developed by IRAP-UPS/CNRS (http://cassis.irap.omp.eu) 

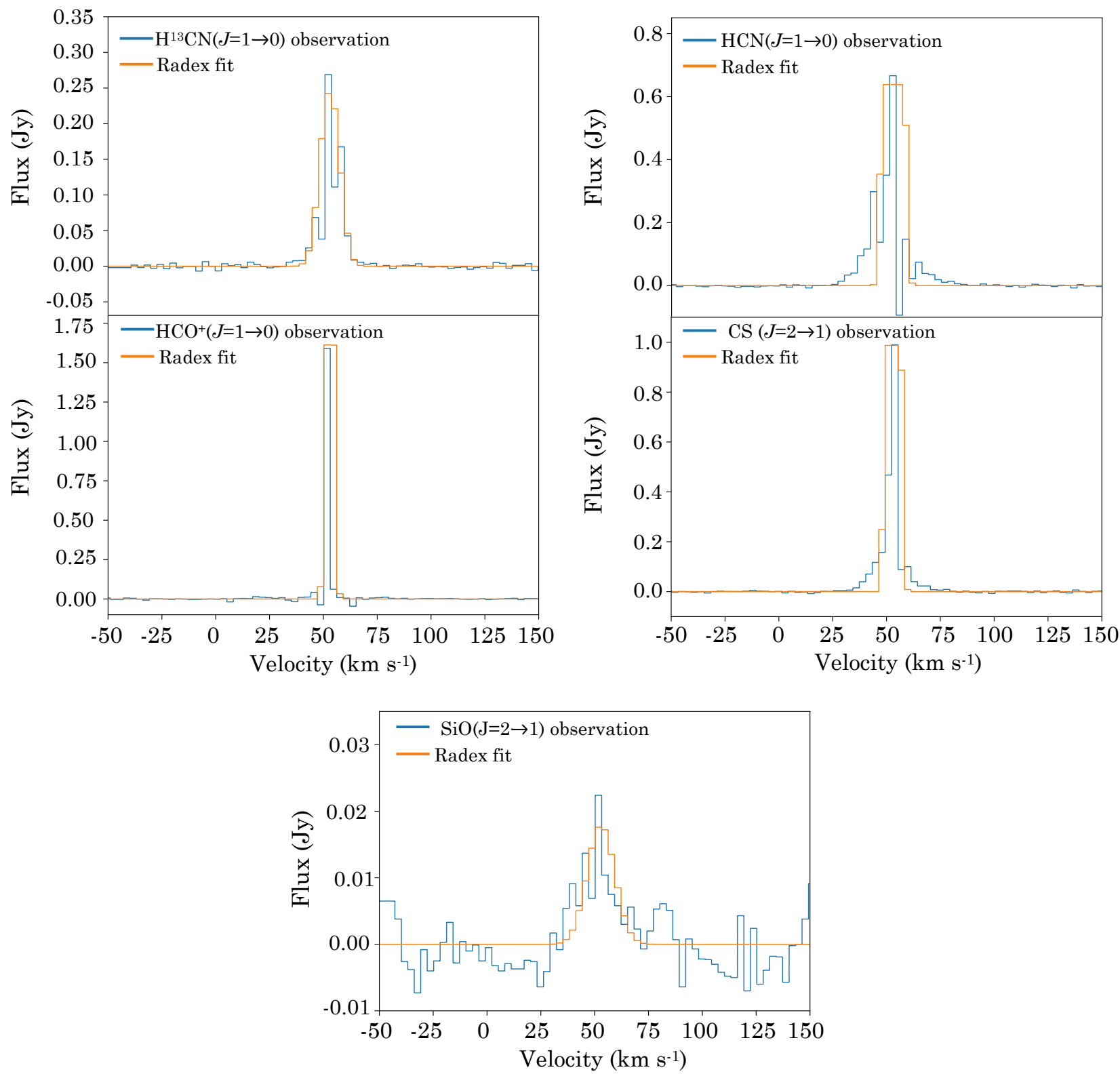

Figure 6. Spectral line profiles of different molecular species detected in G43.89(C) or G43.890-0.784. The blue lines are the ALMA observations and the orange lines are fits to the observations obtained using the RADEX in CASSIS (see Table 6 for the parameters of the fit models).

umn density of $\mathrm{N}\left(\mathrm{H}_{2}\right)=2 \times 10^{24} \mathrm{~cm}^{-2}$, which translates into a volumetric density of $\mathrm{n}\left(\mathrm{H}_{2}\right)=2.6 \times 10^{7} \mathrm{~cm}^{-3}$ and a mass of $\mathrm{M}\left(\mathrm{H}_{2}\right)=220 \mathrm{M}_{\odot}$. The physical parameters of the best fit models are listed in Table 6 . The kinetic temperature of the gas ranges from 16 to $35 \mathrm{~K}$. The gas traced by $\mathrm{HCO}^{+}$is the warmest of all, suggesting that the emission arises closer to a proto-stellar object.

The G43(C) clump does not completely meet the operational definition of a hot molecular core given in Garay \& Lizano (1999, and references therein): size $\lesssim 0.1 \mathrm{pc}$, $\mathrm{T}_{\mathrm{k}}>50 \mathrm{~K}$, and $\mathrm{n}_{\mathrm{e}} \gtrsim 10^{5} \mathrm{~cm}^{-3}$, because the reported temperature is $\lesssim 50 \mathrm{~K}$. Possibly this YSO is in such an early evolutionary stage that the gas has not yet been heated by the YSO or that the dusty molecular core lacks sufficient optical depth to absorb the radiant energy. In an attempt to estimate the evolutionary stage of G43(C), we compare the physical parameters from Table 6 with the chemical models from Nomura \& Millar (2004) assuming a temperaturedependent grain mantle evaporation process. An approximate timescale of $1 \times 10^{4} \mathrm{yr}$ is obtained for a column density of CS $1 \times 10^{16} \mathrm{~cm}^{-2}$.

A timescale of $\sim 6 \times 10^{4} \mathrm{yr}$ is obtained from $\mathrm{HCN}$ (see their Fig. 2(c)). In comparison, the average column densities of some molecular tracers from hot cores given by Li et al. (2015, see their Table 6) are more consistent with a CS timescale between $6 \times 10^{4}$ and $1 \times 10^{5} \mathrm{yr}$, which corresponds to an older stage than for G43(C) by about an order of magnitude. This is in agreement with the chemical evolutionary se- 


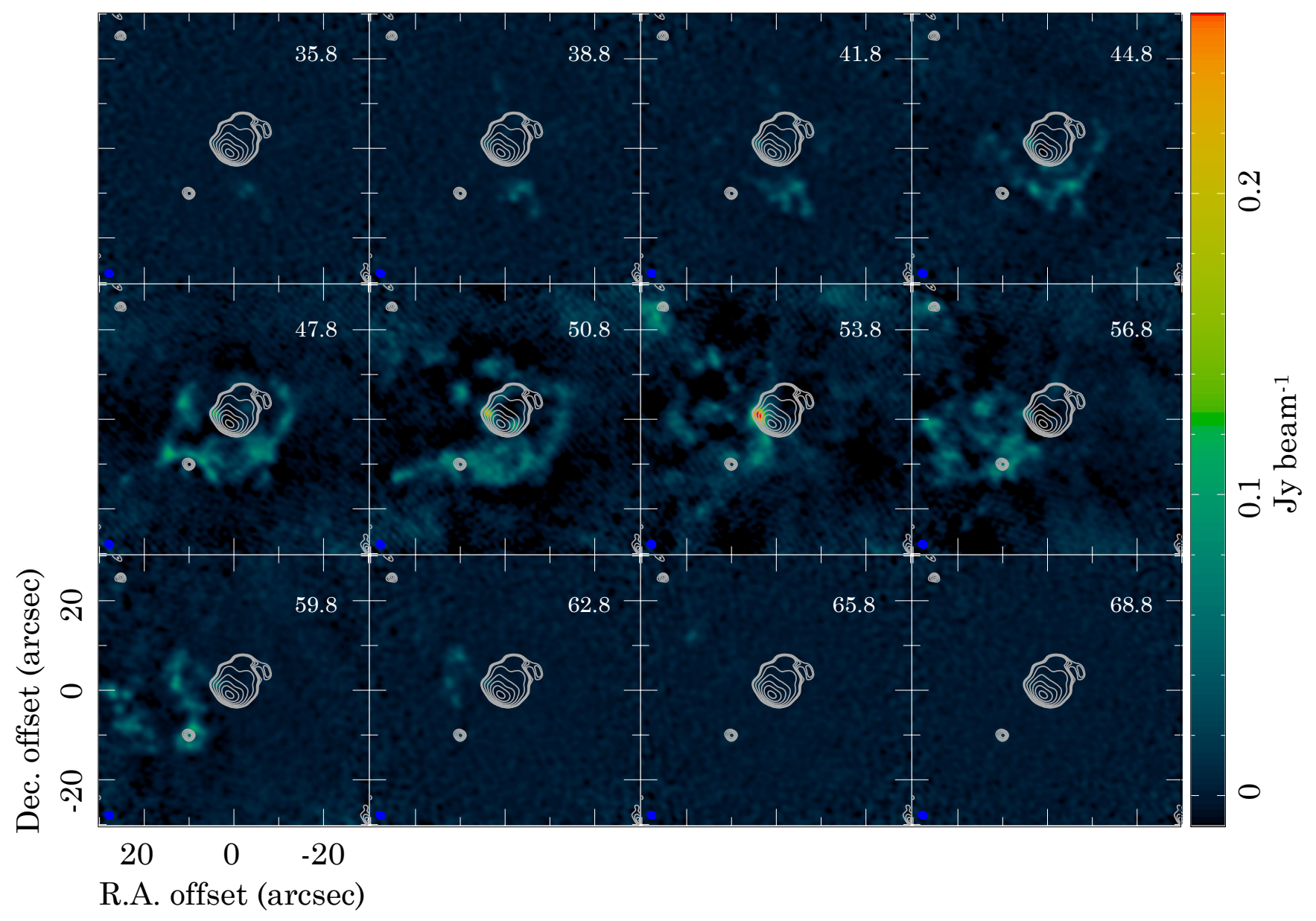

Figure 7. Channel maps of the ALMA CS emission in G43.89-0.78. The colour-map is the CS $(J=2 \rightarrow 1)$ line emission in the vicinity of G43.89-0.78 for individual channel maps. The number in the top-right corner is the velocity of the channel. The white contours represent the continuum emission at $3 \mathrm{~mm}$. The first four contours are 5,6,7 and 8 times the rms of the map (listed on Table 2) and then they increase as $\pm 8 \times \mathrm{rms} \times 2^{i}$ with $i=0,1,2,3 \ldots$. The synthesised beam is indicated with a blue ellipse located in the bottom-left corner of the individual channel maps (see Table 2).

quence proposed by Beuther (2007) for hot molecular cores, where cores at $\sim 30 \mathrm{~K}$ cause some molecules to be released from dust grains (such as CS), but when the temperature of the hot core increases to $\gtrsim 100 \mathrm{~K}$, the $\mathrm{H}_{2} \mathrm{O}$ molecules released also from dust grains generate the enrichment of other molecular tracers and their abundance (e.g., SO).

We also estimated the mass of the clumps G43(N) and G43(S). For a frequency of $86 \mathrm{GHz}$, considering continuum flux densities $\left(S_{v}\right)$, and assuming emission from isothermal, optically thin dust, the gas mass can be determined using (Hildebrand 1983; Gall, Hjorth, \& Andersen 2011):

$\left[\frac{M_{\mathrm{gas}}}{M_{\odot}}\right]=4.8 \times 10^{-23} \frac{g}{B_{v}\left(T_{\text {dust }}\right)}\left[\frac{S_{v}^{\mathrm{N}, \mathrm{S}}}{\mathrm{mJy}}\right]\left[\frac{\kappa_{v}}{\mathrm{~cm}^{2} \mathrm{~g}^{-1}}\right]^{-1}\left[\frac{D}{\mathrm{pc}}\right]^{2}$,

where $g$ is the gas-to-dust ratio, $D$ the adopted distance, $\kappa_{v}^{1.3 m m}$ the dust mass opacity coefficient, and $B_{v}\left(T_{\text {dust }}\right)$ the Planck function for a dust temperature $T_{\text {dust }}$. For a $B_{v}\left(T_{\text {dust }}\right)$ with $\mathrm{T}_{d}=50 \mathrm{~K}$, we derive the clump masses reported in Table 5 . In this calculation, the uncertainty is only referenced to the uncertainty in the adopted value of $\kappa_{v}^{1.3 m m}$; we use $0.015 \mathrm{~cm}^{2} \mathrm{gr}^{-1}$.

\subsubsection{A molecular outflow from the clump $G_{4} 3(C)$ ?}

From the line profiles shown in Fig. 6 it can be seen that some molecular species, such as HCN and CS, exhibit wings with relatively high velocities (spanning a velocity range of $\sim 50 \mathrm{~km} \mathrm{~s}^{-1}$ ). Such wings cannot be fitted with any Gaussian curve. Furthermore, the $\operatorname{CS}(J=2 \rightarrow 1)$ emission in the vicinity of G43.89-0.78 exhibits a NE-SW velocity gradient, which can be seen in Figs. 7 and 8 .

In particular, there is a feature that extends in the NE-SW direction that connects G43(N) with G43(C) and a clump of molecular emission located SW of G43(C). In addition, the velocity gradient of the water masers associated with G43(C) (see Fig. 8) matches the velocity gradient of the molecular emission. This suggests that G43(C) hosts a bipolar molecular outflow that may be the origin of the water masers, as has been observed in other sources (Trinidad 2009). The direction of the outflow is indicated with a broken line in Fig. 8. Further investigations with higher angular resolution, involving proper motion observations, are needed to confirm this idea and to elucidate the nature of this object. 


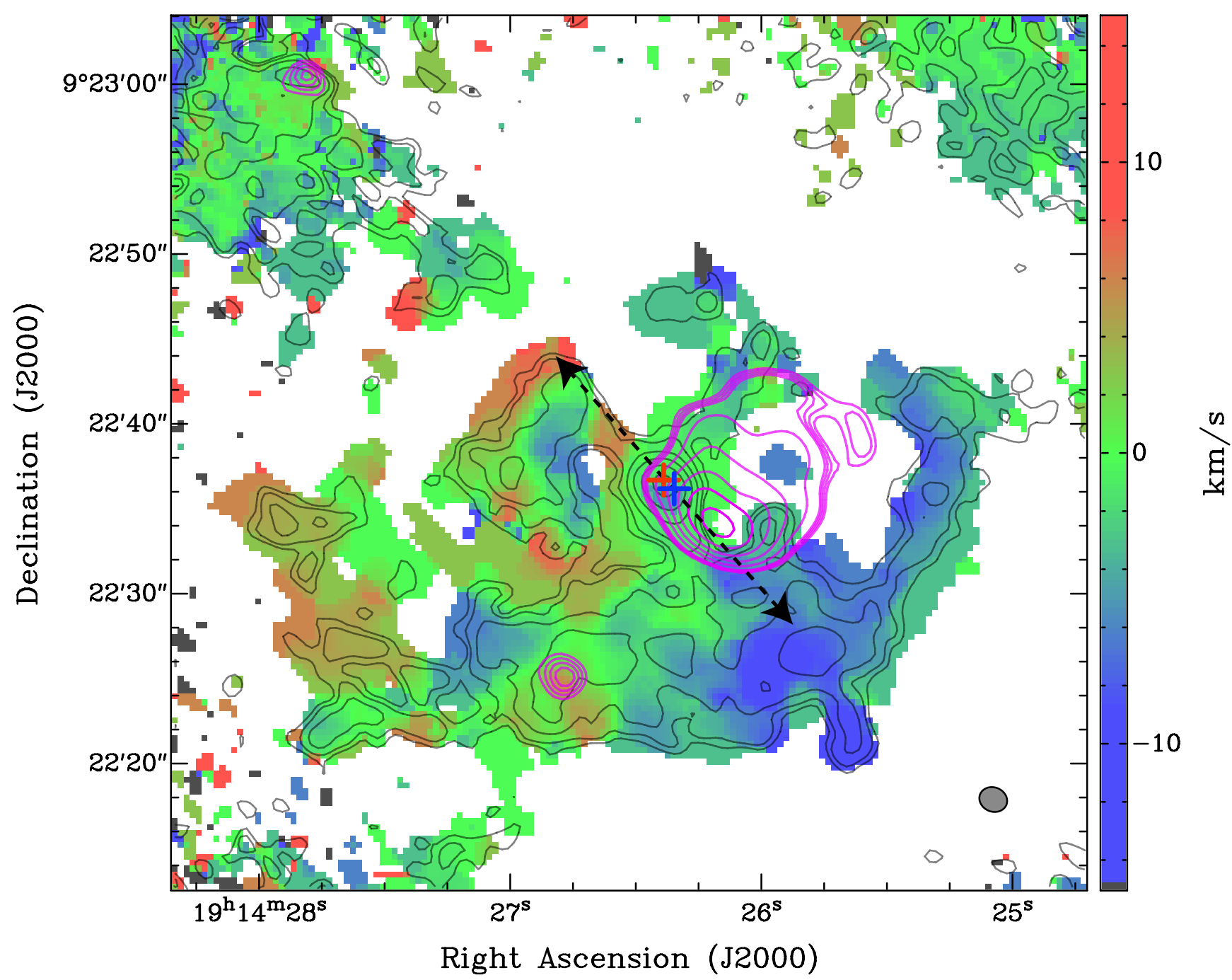

Figure 8. Moment-1 image of the $\operatorname{CS}(J=2 \rightarrow 1)$ line emission in the vicinity of G43.89-0.78. The colour-map is the moment-1 (velocity field) clipped at $0.01 \mathrm{Jy}_{\text {beam }}^{-1}$ (4 times the rms noise level of the channel maps). The black contours indicate the velocity-integrated $\mathrm{CS}(\boldsymbol{J}=2 \rightarrow 1)$ emission (moment- 0 ). The contours increase as $\pm 5 \times \mathrm{rms} \times 1.5^{i}$ with $i=0,1,2,3, \ldots$, where the rms is $0.075 \mathrm{Jy}^{\mathrm{beam}}{ }^{-1} \mathrm{~km} \mathrm{~s}^{-1}$. The magenta contours represent the continuum emission at $3 \mathrm{~mm}$. The first four contours are $5,6,7$ and 8 times the rms of the map (listed on Table 2) and then they increase as $\pm 8 \times \mathrm{rms} \times 2^{i}$ with $i=0,1,2,3 \ldots$. A broken line with arrows indicates the direction of a kinematical feature associated with the clump G43(C) that could be a molecular outflow (see main text).

\section{SUMMARY AND CONCLUSIONS}

(i) The EE associated with G43.89 meets the nominal definition of UC H II +EE regions based on physical parameters: $\mathrm{EM} \sim 10^{4}-10^{5} \mathrm{~cm}^{-6} \mathrm{pc}, \mathrm{n}_{\mathrm{e}} \lesssim 5 \times 10^{2} \mathrm{~cm}^{-3}$, and $\mathrm{M}_{\mathrm{HII}} \sim$ 5 to $10^{3} \mathrm{M}_{\odot}$, therefore it can be added to the final catalogue given in Paper I.

(ii) G43.89-0.78 is confirmed as an UC H II region using high-resolution VLA data at $3.6 \mathrm{~cm}$. It has a dense $\left(2.6 \times 10^{7}\right.$ $\left.\mathrm{cm}^{-3}\right)$, and small $(\sim 2$ " or $0.08 \mathrm{pc})$ nearby molecular clump with mass of $220 \mathrm{M}_{\odot}$. The water masers appear to be spatially coincident with this clump, and may arise in a molecular outflow.

(iii) The low temperature of this clump implies that it is not a hot molecular core, however a timescale of few times $10^{4} \mathrm{yr}$ is estimated by comparison with chemical models.
This value is smaller than the average timescale for typical hot cores by about an order of magnitude.

(iv) G43.890-0.784 is rich in molecular species, and we detect two new radio-continuum clumps, G43.899-0.786 and G43.888-0.787 respectively, located in its vicinity.

\section{ACKNOWLEDGEMENTS}

The National Radio Astronomy Observatory is a facility of the National Science Foundation operated under cooperative agreement by Associated Universities, Inc. This work is based in part on observations made with the Spitzer Space Telescope, which is operated by the Jet Propulsion Laboratory, California Institute of Technology under a contract with NASA. ALMA is a partnership of ESO (representing its member states), NSF (USA), and NINS (Japan), 
together with NRC (Canada), MOST, and ASIAA (Taiwan), and KASI (Republic of Korea), in cooperation with the Republic of Chile. The Joint ALMA Observatory is operated by ESO, AUI/NRAO, and NAOJ. EdlF acknowledges support from Consejo Nacional de Ciencia y Tecnología (CONACyT), Mexico: grant 124449, and CONACyT-SNI exp. 1326. EdelF also thanks Chalmers University of Technology, Onsala Space, Sweden for the support during several research stays. M.A.T. acknowledges support from Universidad de Guanajuato grant DAIP-33/2019. AP acknowledges INAOE/CONACyT financial support for authorship while home-working during the COVID-19 pandemic.

\section{DATA AVAILABILITY}

The data underlying this article are available in:

$$
\text { 1.- ALMA archive }
$$

https://almascience.eso.org/asax/, and can be acces with ALMA\#2015.1.00280.S.

$\begin{array}{ccccr}2 .- & \text { NRAO } & \text { Science } & \text { Data } & \text { Archive } \\ \text { [Karl } & \text { Jansky } & \text { VLA } & \text { Database] } & \text { at }\end{array}$
https://archive.nrao.edu/archive/archiveproject.jsp, and can be accessed with the respective Project (Proposal) Code: AK423

3.- IRSA NASA/IPAC Infrared Science Archive at https://irsa.ipac.caltech.edu/Missions/spitzer.html, and can be accessed by selection of GLIMPSE II (program ID=187), Data Set Identification = ads/sa.spitzer\#0011972096; and MIPSGAL, program IDs 20597 and 30592.

4.- OVRO data will be shared on reasonable request to the corresponding author.

\section{REFERENCES}

Araya E., Hofner P., Churchwell E., Kurtz S., 2002, ApJS, 138, 63

Baudry A., Desmurs J. F., Wilson T. L., Cohen R. J., 1997, A\&A, 325,255

Baug T., Dewangan L. K., Ojha, D. K., Ninan, J. P., 2010, ApJ, 833,85

Benjamin R. A., Churchwell E., Babler B. L., et al., 2003, PASP, 115,953

Beuther H., 2007, Proc. IAU 2, Symp., 237, 148

Bronfman L., Nyman L. A., May J., 1996, A\&A, 11581

Carey S. J., Noriega-Crespo A., Mizuno D. R., et al., 2009, PASP, 121,76

Cesaroni R., Walmsley C. M., Churchwell E., 1992, A\&A, 256, 618

Churchwell E., Babler B. L., Meade M. R., et al., 2009, PASP, 121,213

Churchwell E., Watson D. F., Povich M. S., et al., 2007, ApJ, 670, 428

Churchwell E., Povich M. S., Allen D., et al., 2006, ApJ, 649, 759 Churchwell E., 2002, ARA\&A, 40, 27

Churchwell E., Walmsley C. M., Cesaroni R., 1990, A\&A, 83, 119

Codella C., Cesaroni R., LÃşpez-Sepulcre A., et al., 2010, A\&A, 510, A86

Codella C., Lorenzani A., Gallego A. T., et al., 2004, A\&A, 417, 615

Condon J. J., Cotton W. D., Greisen, E. W., et al., 1998, AJ, 115, 1693 de la Fuente E., Porras A., Trinidad M. A., et al., 2020, MNRAS, 492, 895 (Paper I)

de la Fuente E., Trinidad M. A., Porras A., et al., 2018, Rev. Mex. Astron. Astrofis., 54, 129

de La Fuente E., Kurtz S. E., Kumar M. S. N., et al., 2009a, Astrophys. Space Sci., 7, 167

de La Fuente E., Porras A., Grave J. M. C., et al., 2009b, Rev. Mex. Astron. Astrofis. Conf. Ser., 37, 13

de la Fuente E., 2007, PhD thesis, Departamento de Física, CUCEI, Universidad de Guadalajara, México

Doherty R. M., Puxley P., Doyon R., Brand P. W., 1994, MNRAS, 266, 487

De Pree C. G., Wilner D. J. Goss W. M., 2011, AJ, 142, 177

Dupree A. K., Goldberg L., 1970, ARA\&A, 8, 231

Elitzur M., 1992, ARA\&A, 30, 75

Ellingsen S. P., Shabala S. S., Kurtz S. E., 2005, MNRAS, 357, 1003

Everett J. E., Churchwell, E., 2010, ApJ, 713, 592

Fazio G. G., Hora J. L., Allen L.E., et al., 2004, ApJS, 154, 10

Franco-Hernández R., Rodríguez L. F., 2004, ApJ, 604, L105

Fujita S., Torii K., Tachihara K., Enokiya R., 2019, ApJ, 872, 49

Fukui Y., Ohama A., Hanaoka, N., 2014, ApJ, 780, 36

Furukawa N., Dawson J. R., Ohama A., 2009, ApJ, 696, L115

Gall C., Hjorth J., Andersen A. C., 2011, A\&A Rev., 19, 43

Gálvan-Madrid R., Rodríguez L. F., Ho P. T. P., Keto E., 2008, ApJ, 674, L33

Garay G., Lizano S., 1999, PASP, 111, 1049

García-Segura G., Franco J., 1996, ApJ, 469, 171

Gaume R. A., Goss W. M., Dickel H. R., et al., 1995, ApJ, 438, 776

Habe A., Ohta, K., 1992, PASJ, 44, 203

Hanson M. M., Luhman K. L., Rieke G. H., 2002, ApJS, 138, 35

Hatchell J., Fuller G. A., Millar T. J., et al., 2000, A\&A, 357, 637

Hatchell J., Thompson M.A., Millar T., Macdonald G., 1998, A\&A133, 29

Hildebrand R. H. 1983, QJRAS, 24, 267

Hofner P., Churchwell E., 1996, A\&A, 120, 283

Israel F. P., Habing H. J., de Jong T., 1973, A\&A, 27, 143

Kim K. T., Koo B. C., 2003, ApJ, 596, 362

Kim K. T., Koo B. C., 2002, ApJ, 575, 327

Kim K. T., Koo B. C., 2001, ApJ, 549, 979

Kuchar T. A., Bania T. M., 1990, ApJ, 352, 192

Kurtz S., Franco J., 2002, Rev. Mex. Astron. Astrofis. Conf. Ser, 12,16

Kurtz S., 2000, Rev. Mex. Astron. Astrofis. Conf. Ser, 9, 169

Kurtz S., Cesaroni R., Churchwell E., et al., 2000, Protostars and Planets IV, 299

Kurtz S. E., Watson A. M., Hofner P., Otte B., 1999, ApJ, 514, 23

Kurtz S., Churchwell E., Wood D. O. S., 1994, ApJS, 91, 659

Kuwahara S., Kazufumi T., Norikazu M., et al. 2020, PASJ, doi:10.1093/pasj/psaa017

Li J., Wang J., Zhu Q., et al., 2015, ApJ, 802, 40

Mezger P. G., Henderson A. P., 1967, ApJ, 147, 471

Nagasawa M., Miyama S. M., 1987, Prog. Theor. Phys., 78, 1250

Nomura H., Millar T. J., 2004, A\&A, 414, 409

Ohama A., Kohno M., Hasegawa K., 2018, PASJ70, S45

Ohama, A.; Dawson, J. R.; Furukawa, 2010 ApJ, 709, 975

Olmi L., Cesaroni R., 1999, A\&A, 352, 266 O

Olmi L., Cesaroni R. \& Walmsley C. M., 1993, A\&A, 276, 489

Oster L., 1961, Rev. Mod. Phys., 33, 525

Palau A., Estalella R., Ho P. T. P., et al. 2007, A\&A, 474, 911

Panagia N., Walmsley C. M., 1978, A\&A, 70, 411

Panagia N., 1973, AJ, 78, 929

Rieke G. H., Young E. T., Engelbracht C. W., et al. 2004, ApJS, 154, 25

Robitaille T. P., Meade M. R., Babler B. L., et al., 2008, AJ, 136, 2413 
Robitaille T. P., Whitney B. A., Indebetouw R., et al., 2006, ApJS, 167, 256

Ryle M., Downes D. 1967, ApJ, 148, L17

Sánchez-Monge A., Schilke P., Schmiedeke A., et al., A\&A, 604, A6

Schraml J., Mezger P. G., 1969, ApJ, 156, 269

Schutte A. J., van der Walt D. J., Gaylard M. J., MacLeod G. C., 1993, MNRAS, 261, 783

Scoville N. Z., Sargent A. I., Sanders D. B., et al., 1986, ApJ, 303, 416

Sewiło M., Churchwell E., Kurtz S., et al., 2008, ApJ, 681, 350

Sewiło M., Churchwell E., Kurtz S., Goss W. M., 2004, ApJ, 605, 285

Shepherd, D., Churchwell E., 1996, ApJ, 457, 267

Simpson R. J., Povich M. S., Kendrew S., et al., 2012, MNRAS, 424,2442

Torii K., Enokiya R., Sano H., et al., 2011, ApJ, 738, 46

Torii K., Hasegawa, K.., Hattori, Y., et al., 2015, ApJ, 806, 7

Torii K., Hattori Y., Hasegawa K., et al., 2015, ApJ, 835, 142

Trinidad M. A., Rojas V., Plascencia J. C., et al., 2003, Rev. Mex. Astron. Astrofis., 39, 311

Trinidad, M. A., 2009, Astrophysics and Space Science Proceedings, 13,627

Wenger T.V, Balser D. S., Anderson L. D., Bania T. M., 2018, ApJ, 856, 52

Werner M., Roellig T. L., Low F. J., et al., 2004, ApJS, 154, 1

Wink J.E., Altenhoff W.J., Mezger P.G., 1982, A\&A, 108, 277

Wood D.O.S., Churchwell E., 1989, ApJS, 69, 831

Wouterloot J. G., Brand J., Burton W. B., Kwee K. K., 1990, A\&A, 230, 21.

\section{APPENDIX A: PHYSICAL PARAMETERS (3.6 CM) OF UC H II REGIONS WITH EXTENDED EMISSION}

To obtain the physical parameters of the ionized gas, we assume an isothermal, pure hydrogen, homogeneous, spherically symmetric (radius $r$ and size $\Theta_{\mathrm{S}}$ ), optically thin H II region, with a distance $D$ from the Sun and electronic temperature $\mathrm{T}_{\mathrm{e}}$. The emission measure (EM), electronic density $\left(\mathrm{n}_{\mathrm{e}}\right)$, the mass of the ionized gas $\left(\mathrm{M}_{\mathrm{HII}}\right)$, and the total rate of emission of Lyman continuum photons of the ionizing star $\left(\mathrm{N}_{\mathrm{c}}^{\prime}\right)$ were calculated in the standard way using equations A1 to A4 (Kurtz et al. 1994; Panagia \& Walmsley 1978; Schraml \& Mezger 1969; Oster 1961). The spectral type was obtained using Panagia (1973).

The physical parameters of UC H II +EE regions from Paper I are shown in Table A1. For them, the total rate of emission of Lyman continuum photons and ionizing star type are shown in Paper I. For these UC H II +EE regions, values are: size $\sim 1-4^{\prime}(\sim 1-14 \mathrm{pc}), \mathrm{EM} \sim 10^{4}-10^{5} \mathrm{~cm}^{-6} \mathrm{pc}$, $\mathrm{n}_{\mathrm{e}} \lesssim 5 \times 10^{2} \mathrm{~cm}^{-3}$, and $\mathrm{M}_{\mathrm{HII}} \lesssim 10^{3} \mathrm{M}_{\odot}$. The values reported by Kim \& Koo (2001) at $20 \mathrm{~cm}$ are: size $\sim 1-13^{\prime}(\sim 3-19 \mathrm{pc})$, $\mathrm{EM} \sim 10^{4} \mathrm{~cm}^{-6} \mathrm{pc}, \mathrm{n}_{\mathrm{e}} \lesssim 10^{2} \mathrm{~cm}^{-3}$, and $\mathrm{M}_{\mathrm{HII}} \sim 10^{2}$ to $10^{3}$ $\mathrm{M}_{\odot}$. Combining both samples, we confirm UC H II +EE regions are H II regions with these values: sizes $\sim 1-10^{\prime}(\sim$ $1-20 \mathrm{pc}), \mathrm{EM} \sim 10^{4}-10^{5} \mathrm{~cm}^{-6} \mathrm{pc}, \mathrm{n}_{\mathrm{e}} \lesssim 5 \times 10^{2} \mathrm{~cm}^{-3}$, and $\mathrm{M}_{\mathrm{HII}} \sim 5$ to $10^{3} \mathrm{M}_{\odot}$.

Table 1 of Kurtz \& Franco (2002) summarizes the physical parameters of several types of H II regions. Updating with information from the $3.6 \mathrm{~cm}$ observations, we show an adaptation of this table, including $\mathrm{UC} \mathrm{H}$ II +EE regions in Table A2.

$$
\begin{aligned}
&\left(\frac{\mathrm{n}_{\mathrm{e}}}{\mathrm{cm}^{-3}}\right)=7.8 \times 10^{3}\left(\frac{v}{4.9 \mathrm{GHz}}\right)^{0.05}\left(\frac{\mathrm{S}_{v}}{\mathrm{mJy}}\right)^{0.5}\left(\frac{\mathrm{T}_{\mathrm{e}}}{10^{4} \mathrm{~K}}\right)^{0.175} \\
& \times\left(\frac{\Theta_{\mathrm{s}}}{\operatorname{arcsec}}\right)^{-1.5}\left(\frac{\mathrm{D}}{\mathrm{kpc}}\right)^{-0.5},( \\
&\left(\frac{\mathrm{EM}}{\mathrm{cm}^{-3}}\right)=4.4 \times 10^{5}\left(\frac{v}{4.9 \mathrm{GHz}}\right)^{0.1}\left(\frac{\mathrm{S}_{v}}{\mathrm{mJy}}\right)\left(\frac{\mathrm{T}_{\mathrm{e}}}{10^{4} \mathrm{~K}}\right)^{0.35} \\
&\left(\frac{\mathrm{M}_{\mathrm{HII}}}{\mathrm{M}_{\odot}}\right)=3.7 \times 10^{-5}\left(\frac{\Theta_{\mathrm{s}}}{4.9 \mathrm{GHz}}\right)^{-2}, \\
& \operatorname{arcsec}^{0.05}\left(\frac{\mathrm{S}_{v}}{\mathrm{mJy}^{\prime}}\right)^{0.5}\left(\frac{\mathrm{T}_{\mathrm{e}}}{10^{4} \mathrm{~K}}\right)^{0.175} \\
&\left(\frac{\mathrm{N}_{\mathrm{c}}^{\prime}}{\mathrm{s}^{-1}}\right) \geqslant 8.04 \times 10^{46}\left(\frac{\mathrm{T}_{\mathrm{e}}}{\mathrm{K}}\right)^{-0.85}\left(\frac{\mathrm{r}}{\mathrm{ac}}\right)^{3}\left(\frac{\mathrm{D}}{\mathrm{arcsec}^{-3}}\right)^{2.5},
\end{aligned}
$$

This paper has been typeset from a $\mathrm{T}_{\mathrm{E} X} / \mathrm{LAT}_{\mathrm{E}} \mathrm{X}$ file prepared by the author. 
14 Eduardo de la Fuente et al.

Table A1. Physical Parameters of the UC H II +EE regions reported in Table 7 of Paper I, using RC observations at $3.6 \mathrm{~cm}{ }^{\text {a }}$.

\begin{tabular}{lcccccr}
\hline $\begin{array}{l}\text { Source } \\
\text { Name }\end{array}$ & $\begin{array}{c}\mathrm{S}_{v}{ }^{\mathrm{a}} \\
(\mathrm{Jy})\end{array}$ & $\begin{array}{c}\text { Source }^{\mathrm{b}} \\
\left.\text { Size }^{(}\right)\end{array}$ & $\begin{array}{c}\text { Source }^{\mathrm{b}} \\
\text { Size }(\mathrm{pc})\end{array}$ & $\begin{array}{c}\mathrm{EM} \\
\left(10^{4} \mathrm{~cm}^{-6} \mathrm{pc}\right)\end{array}$ & $\begin{array}{c}\mathrm{n}_{\mathrm{e}} \\
\left(\mathrm{cm}^{-3}\right)\end{array}$ & $\begin{array}{r}\mathrm{M}_{\mathrm{H} \mathrm{II}} \\
\left(\mathrm{M}_{\odot}\right)\end{array}$ \\
\hline G05.48-0.24 & 1.18 & 2.45 & 3.10 & 2.50 & 40.80 & 1799.55 \\
G05.97-1.17 & 8.06 & 2.65 & 2.08 & 14.80 & 218.40 & 81.96 \\
G10.30-0.15 & 5.80 & 2.45 & 4.28 & 12.50 & 139.80 & 454.96 \\
G12.21-0.10 & 1.30 & 3.46 & 13.59 & 1.40 & 26.30 & 2745.07 \\
G18.15-0.28 & 4.20 & 2.83 & 3.46 & 6.76 & 114.50 & 197.04 \\
G19.60-0.23 & 4.33 & 1.26 & 1.28 & 35.18 & 428.80 & 37.68 \\
G23.44-0.21 & 1.16 & 2.00 & 5.24 & 3.74 & 69.20 & 413.54 \\
G23.71+0.17 & 1.49 & 2.00 & 5.18 & 4.80 & 78.90 & 455.77 \\
G25.71+0.04 & 0.68 & 3.46 & 9.36 & 0.73 & 22.90 & 782.00 \\
G28.20-0.05 & 0.48 & 1.50 & 3.97 & 2.75 & 68.20 & 177.62 \\
G31.39-0.25 & 0.65 & 1.94 & 5.02 & 2.23 & 54.50 & 287.59 \\
G35.20-1.74 & 11.31 & 2.00 & 1.92 & 3.65 & 112.80 & 33.24 \\
G37.55-0.11 & 0.93 & 2.00 & 5.76 & 3.00 & 59.10 & 469.90 \\
G37.87-0.40 & 4.11 & 1.00 & 2,71 & 53.00 & 362.50 & 298.72 \\
G45.07+0.13 & 0.73 & 1.00 & 1.75 & 9.42 & 190.20 & 42.09 \\
G45.12+0.13 & 1.80 & 1.50 & 2.62 & 10.32 & 162.60 & 121.42 \\
G45.45+0.06 & 4.50 & 2.00 & 3.49 & 14.51 & 166.90 & 295.57 \\
G54.10-0.06 & 0.52 & 2.45 & 5.63 & 1.12 & 36.50 & 270.99 \\
G60.88-0.13 & 0.49 & 1.73 & 1.11 & 2.11 & 113.10 & 6.39 \\
G77.96-0.01 & 0.95 & 2.45 & 3.14 & 2.04 & 66.10 & 84.80 \\
G111.28-0.66 & 0.30 & 2.50 & 1.82 & 0.62 & 47.80 & 11.95 \\
\hline
\end{tabular}

${ }^{a}$ We use the VLA conf. D data for all sources except G60.88-0.13 (conf. C). See Paper I for details. In this computing, we assume the canonical value for electron temperatures of $1 \times 10^{4} \mathrm{~K}$ for all sources. Distances are reported on Table 1 of Paper I. The ionizing photon rates and spectral types are shown in Table 7 of Paper I.

${ }^{\mathrm{b}}$ Size $=\sqrt{\Theta_{x} \Theta_{y}}$. The values of $\Theta_{x}$ and $\Theta_{y}$ were obtained from Table 5 of Paper I.

Table A2. Physical parameters of H II regions from radio observations. The ultracompact H II region with extended emission (UC H II +EE ) category is added for the first time using $3.6 \mathrm{~cm}$ data.

\begin{tabular}{|c|c|c|c|c|}
\hline $\begin{array}{l}\text { Class of H II } \\
\text { region }\end{array}$ & $\begin{array}{l}\text { Size } \\
(\mathrm{pc})\end{array}$ & $\begin{array}{c}\mathrm{EM} \\
\left(\mathrm{cm}^{-6} \mathrm{pc}\right)\end{array}$ & $\begin{array}{c}\mathrm{n}_{\mathrm{e}} \\
\left(\mathrm{cm}^{-3}\right)\end{array}$ & $\begin{array}{c}\mathrm{M}_{\mathrm{H} \text { II }} \\
\mathrm{M}_{\odot}\end{array}$ \\
\hline Hypercompact & $\sim 0.003$ & $\gtrsim 10^{10}$ & $\gtrsim 10^{6}$ & $\sim 10^{-3}$ \\
\hline Ultra & $\lesssim 0.1$ & $\gtrsim 10^{7}$ & $\gtrsim 10^{4}$ & $\sim 10^{-3}$ \\
\hline Comp & $\lesssim 0.5$ & $\gtrsim 10^{7}$ & $\gtrsim 5 \times 10^{3}$ & $\sim 1$ \\
\hline $\mathrm{UC} \mathrm{H} \mathrm{II}+\mathrm{EE}$ & $\widetilde{1}-20$ & $10^{4}-10^{5}$ & $\lesssim 5 \times 10^{2}$ & $5-10^{3}$ \\
\hline Classical & $\sim 10$ & $\sim 10^{2}$ & $\sim 100$ & $\sim 10^{5}$ \\
\hline Giant & $\sim 100$ & $\sim 5 \times 10^{5}$ & $\sim 10$ & $10^{3}-10^{6}$ \\
\hline Supergiant & $\gtrsim 100$ & $\sim 10^{5}$ & $\sim 10$ & $10^{6}-10^{8}$ \\
\hline
\end{tabular}

